\title{
Conjugates, Filters and Quantum Mechanics
}

\author{
Alexander Wilce \\ Department of Mathematics and Computer Science, Susquehanna University \\ July 2, 2019
}

The Jordan structure of finite-dimensional quantum theory is derived, in a conspicuously easy way, from a few simple postulates concerning abstract probabilistic models (each defined by a set of basic measurements and a convex set of states). The key assumption is that each system $A$ can be paired with an isomorphic conjugate system, $\bar{A}$, by means of a non-signaling bipartite state $\eta_{A}$ perfectly and uniformly correlating each basic measurement on $A$ with its counterpart on $\bar{A}$. In the case of a quantummechanical system associated with a complex Hilbert space $\mathcal{H}$, the conjugate system is that associated with the conjugate Hilbert space $\mathcal{H}$, and $\eta_{A}$ corresponds to the standard maximally entangled EPR state on $\mathcal{H} \otimes \overline{\mathcal{H}}$. A second ingredient is the notion of a reversible filter, that is, a probabilistically reversible process that independently attenuates the sensitivity of detectors associated with a measurement. In addition to offering more flexibility than most existing reconstructions of finite-dimensional quantum theory, the approach taken here has the advantage of not relying on any form of the "no restriction" hypothesis. That is, it is not assumed that arbitrary effects are physically measurable, nor that arbitrary families of physically measurable effects summing to the unit effect, represent physically accessible observables. (An appendix shows how a version of Hardy's "subpace axiom" can replace several assumptions native to this paper, although at the cost of disallowing superselection rules.)

\section{Introduction and Overview}

A number of recent papers, notably $[11,14,20,25,28]$, have succeeded in deriving the mathematical apparatus of finite-dimensional quantum mechanics (henceforth: QM) from various packages of broadly operational, probabilistic, or information-theoretic assumptions. These assumptions are, however, rather strong, and the derivations themselves are not trivial. This paper aims at a slightly broader target, and finds it much easier to hit.

Specifically, the Jordan structure of finite-dimensional quantum theory is derived, in a conspicuously easy way, from a few simple principles. This still brings us within hailing distance of standard QM, owing to the classification theorem for finite-dimensional formally real Jordan algebras as direct sums of real, complex and quaternionic quantum systems, spin factors ("bits" of arbitrary dimension), and the exceptional Jordan algebra [22]. In contrast, all of the cited reconstructions make use of strong axioms that rule out real and quaternionic systems, and even complex quantum systems with superselection rules, more or less by fiat. Since there are good arguments for taking real and quaternionic quantum systems seriously (see [4] for a forceful argument in this direction), it is of interest to have an axiomatic scheme that accommodates them.

Alexander Wilce: wilce@susqu.edu 
I shall have more to say on this point below.

Correlation in quantum mechanics The approach taken here begins with a simple and wellknown observation about finite-dimensional quantum systems. Let $\mathcal{H}$ be an $n$-dimensional complex Hilbert space ${ }^{1}$, representing a finite-dimenional quantum system. Recall that the conjugate Hilbert space, $\overline{\mathcal{H}}$, is the same abelian group, but endowed with the scalar multiplication $(c, x) \mapsto \bar{c} x$ (where the scalar multiplication on the right is that in $\mathcal{H}$, and $\bar{c}$ is the complex conjugate of $c \in \mathcal{C}$ ), and with inner product $(x, y) \mapsto\langle y, x\rangle$. It is customary to write $\bar{x}$ for the vector $x \in \mathcal{H}$, regarded as a vector in $\overline{\mathcal{H}}$, so that $c \bar{x}=\bar{c} x$, or, equivalently, $\overline{c x}=\bar{c} \bar{x}$. The inner product on $\mathcal{H}$ is then given by $\langle\bar{x}, \bar{y}\rangle=\langle y, x\rangle=\overline{\langle x, y\rangle} .{ }^{2}$

Suppose now that $W$ is any density operator on $\mathcal{H}$, with spectral decomposition $W=$ $\sum_{x \in E} \lambda_{x} p_{x}$ for some orthonormal basis $E$, where $p_{x}$ is the rank-one projection associated with a unit vector $x \in E$. Then $W$ is the marginal, or reduced state, of the pure bipartite state

$$
\Psi_{W}:=\sum_{x \in E} \lambda_{x}^{1 / 2} x \otimes \bar{x} \in \mathcal{H} \otimes \overline{\mathcal{H}}
$$

The fact that mixed quantum-mechanical states arise in this way as marginals of pure states on larger systems is the starting point for the reconstruction of QM in [11]. Here, we focus instead on the correlational features of $\Psi_{W}$. A straightforward calculation shows that if $a, b$ are any two operators on $\mathcal{H}$, then

$$
\left\langle(a \otimes \bar{b}) \Psi_{W}, \Psi_{W}\right\rangle=\operatorname{Tr}\left(W^{1 / 2} a W^{1 / 2} b\right) .
$$

In particular, if $a$ and $b$ commute with $W$, then we have

$$
\left\langle(a \otimes \bar{b}) \Psi_{W}, \Psi_{W}\right\rangle=\operatorname{Tr}(W a b) .
$$

It follows that the state $\Psi_{W}$ perfectly correlates any projection-valued observable that commutes with $W$, with its counterpart on $\overline{\mathcal{H}}$ : if $a$ and $b$ are mutually orthogonal projections, both commuting with $W$, then the joint probability of observing $a$ and $\bar{b}$ is $\left\langle\Psi_{W}, a \otimes \bar{b}\right\rangle=\operatorname{Tr}(W a b)=0$, while the joint probability of $a$ and $\bar{a}$ is $\left\langle\Psi_{W}, a \otimes \bar{a}\right\rangle=\operatorname{Tr}(W a)$. Where $a=p_{x}$ is the rank-one projection associated with a unit vector $x$, this means that the conditional state of the conjugate system, given a measurement result $x$ on the system corresponding to $\mathcal{H}$, is the "collapsed" state corresponding $\bar{x}$. In effect, the entangled state $\Psi_{W}$ allows the conjugate system to retain a record of the measurement result on the first system - even though no signal need have passed between the two.

A striking special case arises where $W=\frac{1}{n} \mathbf{1}$, the maximally mixed state: in this case, $\Psi_{W}$ is the "EPR" state

$$
\Psi=\frac{1}{\sqrt{n}} \sum_{x \in E} x \otimes \bar{x}
$$

the expansion being independent of the choice of the orthonormal basis $E$. As every observable commutes with $W, \Psi$ perfectly, and uniformly, correlates every observable on $\mathcal{H}$ with its counterpart on $\overline{\mathcal{H}}$. Thus, if we imagine that the system corresponding to $\mathcal{H}$ is controlled by Alice and that corresponding to $\overline{\mathcal{H}}$, by Bob, then if Alice and Bob happen to make the same measurement,

\footnotetext{
${ }^{1} \mathrm{~A}$ word on notation: I follow the mathematicians' convention that a complex inner product $\langle$,$\rangle is conjugate-$ linear in the second, rather than the first argument. Thus, in terms of Dirac notation, $\langle x, y\rangle=\langle y \mid x\rangle$.

${ }^{2}$ One can think of $\overline{\mathcal{H}}$ as the space of bras $\langle x|$ corresponding to the kets $|x\rangle \in \mathcal{H}$, but I prefer to avoid this representation, since I want to stress the idea that $\overline{\mathcal{H}}$ represents a quantum system in its own right. Thus, using Dirac notation we might write $|\bar{x}\rangle=\langle x|$.
} 
they are bound to obtain the same result, with uniform probability $1 / n$. Notice, also, that by (2) we have

$$
\langle(a \otimes \bar{b}) \Psi, \Psi\rangle=\frac{1}{n} \operatorname{Tr}(a b)
$$

for all observables $a$ and $b$, so the state $\Psi$ in some sense explains the normalized trace inner product.

Correlation in General Probabilistic Theories These correlational features make sense in a much more general setting. As explained in more detail below, a probabilistic model is characterized by a set of basic measurements or experiments, and a convex set of states, with each state $\alpha$ assigning a probability $\alpha(x)$ to every outcome $x$ of every basic measurement. Given two such models $A$ and $B$, a bipartite state $\omega$ on $A$ and $B$ is an assignment of joint probabilities $\omega(x, y)$ to all outcomes $x$ and $y$ of basic $A$ - and $B$-measurements, respectively, having well defined conditional and marginal (reduced) probability weights corresponding to states of $A$ and $B$.

We now impose some restrictions on the probabilistic models under consideration. First, we require all state spaces to be finite-dimensional (we are, after all, only attempting to recover finite-dimensional QM). Secondly, we require that models be uniform, in the sense that

(i) all basic measurements have a common, finite number of outcomes, $n$, called the rank of $A$; and

(ii) there exists a maximally mixed state, $\rho$, defined by $\rho(x)=1 / n$ for all basic measurement outcomes $x$

These conditions are satisfied by finite-dimensional quantum-mechanical models, including those involving superselection sectors, provided that we restrict attention to maximal observables, i.e., those consisting of rank-one projections. More generally, condition (i) is reasonable if we think of basic measurements as maximally informative, so that each has the largest possible number of outcomes, and cannot be further refined. Given condition (i), the maximally-mixed state is welldefined mathematically, so in (ii), we are only requiring that it count as a physically accessible state.

The following is a direct translation of the correlational features discussed above for quantummechanical systems, into the language of probabilistic models.

Definition 1. A conjugate of a (uniform) probabilistic model $A$ is a model $\bar{A}$, together with an isomorphism $\gamma$ taking each basic measurement outcome $x$ of $A$ to an outcome $\bar{x}:=\gamma(x)$ of $\bar{A}$, such that

(a) Every state $\alpha$ of $A$ is the marginal of some state $\omega$ on $A$ and $\bar{A}$ (in general, depending on $\alpha$ ) correlating some basic measurement $E$ of $A$ with its counterpart on $\bar{A}$ so that for all $x \in E$,

$$
\omega(x, \bar{x})=\alpha(x)
$$

so that $\omega(x, \bar{y})=\alpha(x) \delta_{x, y}$.

(b) The maximally mixed state $\rho$ arises as the marginal of a bipartite state $\eta_{A}$ uniformly correlating every basic measurement with its counterpart, in the sense that

$$
\eta_{A}(x, \bar{x})=\frac{1}{n}
$$

for all basic measurement outcomes $x$, where $n$ is the rank of $A$.

Evidently, in the quantum-mechanical case, where $\alpha$ corresponds to a density operator $W$, the state $\Psi_{W}$ supplies the correlating state $\omega$, while the bipartite state $\eta$ corresponds to the EPR state $\Psi_{\frac{1}{n} 1}=\Psi$. 
Mathematically, the existence of a conjugate system has affinities with the purification postulate of [11], though we do not require the correlating bipartite state $\omega$ above to be pure. Physically, a conjugate system $\bar{A}$ allows for the formation of records of the outcomes of measurements on $A$ in causally separated systems, exactly as in the quantum case. Condition (a) above simply requires that, for every state $\alpha$, there be at least one basic measurement on $A$ that can be thus recorded and later "read off" by performing the corresponding measurement on $\bar{A}$. Condition (b) requires that, where $A$ is in the maximally mixed state, it be possible to record every basic measurement in this way.

From correlation to Jordan algebras Remarkably little is required, beyond the existence of a conjugate, to secure a representation of $A$ in terms of a formally real Jordan algebra. This depends on a classic mathematical result, the Koecher-Vinberg Theorem [18]. A finite-dimensional ordered vector space $\boldsymbol{E}$ with positive cone $\boldsymbol{E}_{+}$is self-dual if it carries an inner product such that $a \in \boldsymbol{E}_{+}$ iff $\langle a, b\rangle \geq 0$ for all $b \in \boldsymbol{E}_{+}$. If the group of invertible linear mappings $\boldsymbol{E} \rightarrow \boldsymbol{E}$ carrying $\boldsymbol{E}_{+}$onto itself acts transitively on the interior of the cone $\boldsymbol{E}_{+}$, then $\boldsymbol{E}$ is said to be homogeneous. The Koecher-Vinberg Theorem asserts that if $\boldsymbol{E}$ is both homogeneous and self-dual, it can be endowed with a formally real Jordan structure for which $\boldsymbol{E}_{+}=\left\{a^{2} \mid a \in \boldsymbol{E}\right\}$.

Any probabilistic model $A$ gives rise in a natural way to two ordered vector spaces: a space $\mathbf{V}(A)$, generated by $A$ 's states, and a space $\mathbf{E}(A) \leq \mathbf{V}(A)^{*}$ generated by evaluation functionals $\widehat{x}: \alpha \mapsto \alpha(x)$ associated with basic measurement outcomes $x$. Since we are assuming that the state space is finite dimensional, both of the spaces $\mathbf{E}(A)$ and $\mathbf{V}(A)$ are also finite dimensional, and it is easy to see that they have the same dimension. If we can show that $\mathbf{E}(A)$ is homogeneous and self-dual, then the Koecher-Vinberg Theorem will provide a formally real (equivalently, euclidean) Jordan structure on $\mathbf{E}(A)$ for which the cone of squares coincides with $\mathbf{E}(A)_{+}$.

Call a probabilistic model $A$ sharp if, for every basic measurement outcome $x$, there is a unique state $\delta_{x}$ with $\delta_{x}(x)=1$. Physically, this is a way of saying that basic measurements are maximally informative: if we can predict the outcome with certainty, we know the system's state exactly.

Theorem 1. Suppose $A$ is sharp and has a conjugate. Then the state $\eta_{A}$ gives rise to a selfdualizing inner product on $\mathbf{E}(A)$, with respect to which $\mathbf{E}(A)$ and $\mathbf{V}(A)$ are isomorphic as ordered vector spaces.

It follows that if $\mathbf{V}(A)$ is homogeneous, so is $\mathbf{E}(A)$, whence, by the Koecher-Vinberg Theorem, the latter carries a formally real Jordan structure. But the homogeneity of $\mathbf{V}(A)$ has a direct physical interpretation: it says that for every non-singular state - that is, every state $\alpha$ with $\alpha(x)>0$ for every basic measurement outcome - there exists a probabilistically reversible process $T$ - defined below, but, roughly, one that can be reversed by another process with non-zero probability - such that $T(\rho)=r \alpha$ where $r \in[0,1]$. In other words, every non-singular state can be prepared, up to normalization, by applying a probabilistically reversible process to the maximally mixed state.

In fact, it is enough to assume less. By a filter for a basic measurement with outcome-set $E$, I mean a process $\Phi$ - that is, a positive linear mapping $\Phi: \mathbf{V}(A) \rightarrow \mathbf{V}(A)$ - that independently attenuates the reliability of each outcome $x \in E$, so that for every state $\alpha, \Phi(\alpha)(x)=t_{x} \alpha(x)$ for some constant $t_{x}$ (independent of $\alpha$ ). If we think of basic measurement outcomes as detectors, the existence of such filters, with arbitrary coefficients, is plausible; in standard QM, not only do they exist but, if $t_{x}>0$ for every $x \in E$, then $\Phi$ can be chosen to be probabilistically reversible. Where a probabilistic model $A$ shares this feature, I will say that $A$ has arbitrary reversible filters.

Corollary 1. Suppose that $A$ is sharp, has a conjugate, and has arbitrary reversible filters. Then $\mathbf{E}(A)$ is homogeneous and self-dual. 
If we adopt a stronger assumption about filters, we can weaken the requirement that $A$ have a conjugate, and eliminate entirely the hypothesis that $A$ is sharp. Let us say that $A$ has a weak conjugate if the maximally mixed state $\rho$ is the marginal of a uniformly correlating state $\eta_{A}$ on $A$ and $\bar{A}$, as in condition (b) in Definition 1, but not assuming that every state is the marginal of a correlating state, i.e., not assuming condition (a). That is, we require only that there exist a joint state on two copies of $A$ - an analogue of the EPR state - in which, if the same measurement is performed on each copy, the results are guaranteed to be the same, but are otherwise completely random.

By applying the filter $\Phi$ to the system $A$, and then computing the canonical bipartite state $\eta_{A}$, we obtain a new bipartite state. Equally, we could begin by applying the counterpart of $\Phi$ to the conjugate system, obtaining another bipartite state. If these two bipartite states are in fact the same, we say that $\Phi$ is symmetric.

Corollary 2. Let $A$ have a weak conjugate. If every non-singular state of $A$ can be prepared, up to normalization, from the maximally mixed state by a symmetric, reversible filter, then $\mathbf{E}(A)$ is homogeneous and self-dual.

The proofs of these results are all quite short and straightforward. In summary, we recover euclidean Jordan algebras from either of two distinct, but related, sets of assumptions about physical systems represented by uniform, finite-dimensional probabilistic models:

(a) systems are sharp;

(b) Systems have conjugates; and

(c) Systems have arbitrary reversible filters.

Alternatively, and more compactly:

$\left(\mathrm{b}^{\prime}\right)$ Systems have weak conjugates;

$\left(c^{\prime}\right)$ All non-singular states can be prepared by reversible symmetric filters

Any euclidean Jordan algebra gives rise to a probabilistic model in which basic measurements correspond to Jordan frames, i.e., sets $\left\{e_{i}\right\}$ of minimal idempotents satisfying $e_{i} \bullet e_{j}=0$ for $i \neq j$, and $\sum_{i} e_{i}=\mathbf{1}$, where $\mathbf{1}$ is the Jordan unit. (In standard QM, these would correspond to maximally fine-grained projective measurements.) In Appendix A, it is shown that any such model satisfies all of the assumptions above and, conversely, if $A$ satisfies either package of assumptions, the Jordan product on $\mathbf{E}(A)$ can be chosen so that the set of basic measurements is precisely the set of Jordan frames. Thus, these two sets of assumptions are in fact equivalent, and exactly characterize this class of euclidean Jordan-algebraic probabilistic models.

There is actually a third possibility, in which condition (b) in the definition of a conjugate is replaced by a rather weak symmetry assumption and a version of Hardy's subspace axiom [20]. Again, the resulting package of assumptions is satisfied by, and hence, characterizes, Jordan models. The details are spelled out in Appendix B.

Other reconstructions of QM The approach of this paper offers some significant advantages over the reconstructions of quantum mechanics cited earlier. First, it is simply easier, in the sense that our results are obtained with less mathematical effort. (This, notwithstanding the length 
of this paper, which owes to the inclusion of many details intended to make the paper easier to follow.) ${ }^{3}$

Secondly, it rests on fewer and (arguably) simpler assumptions. Certainly, the second package of asumptions (that is, $\left(\mathrm{b}^{\prime}\right)$ and $\left(\mathrm{c}^{\prime}\right)$ above) is smaller than anything found in earlier reconstructions of QM. Other reconstructions tend to impose strong constraints on subsystems, in effect assuming that every face of the state space corresponds to the state space of a "sub-system", satisfying the remaining axioms. Nothing like this is needed here (though, as mentioned above, if one finds a such a "subspace axiom" compelling, it can be put to good use in the present approach; see Appendix B for details). A related assumption, also used in several of the cited papers, is that all systems having the same "information capacity" - the maximal number of states sharply distinguishable by single-shot measurement - are isomorphic. The present approach entirely avoids such an assumption. It also does without the assumption, commonly called the no-restriction hypothesis [21], used in [25] for bits, that all mathematically possible effects — that is, affine functionals assigning probabilities to states - correspond to physically accessible measurement results. More recently, the interesting paper [7] derives the same Jordan-algebraic structure arrived at here, but in a different way. In addition to a strong symmetry postulate, this paper assumes a weak form of the no restriction hypothesis, namely, that all finite sets of "allowed" effects that sum to the unit effect (the effect identically 1 on all states) correspond to accessible measurements, along with a kind of spectral decomposition for states. Here, we manage without any form of no-restriction assumption, and a spectral decomposition for states is derived, rather than postulated.

Finally, all of the earlier reconstructions of QM cited above assume some form of local tomography. This is the doctrine that the state of a bipartite system is determined by the joint probabilities it assigns to outcomes of measurements on the two component systems. This principle has a certain intuitive appeal; moreover, it is well known, and easy to see on dimensional grounds, that among finite-dimensional real, complex and quaternionic quantum mechanics, only in the complex version are composites locally tomographic.

More generally [19, 8], the only probabilistic theory in which systems correspond to Jordan models and composite systems are locally tomographic, and which includes at least one system having the structure of a qubit, is finite-dimensional complex quantum mechanics. Thus, if one insists on local tomography, it can be added to the list of assumptions discussed above, and leads to standard, complex QM (with superselection rules). One should perhaps not rush to embrace local tomography as a universal principle, however. The very fact that it excludes real and quaternionic quantum theory suggests that it is too strong. There are natural ways of representing complex Hilbert spaces in terms of real or quaternionic ones, and vice versa ${ }^{4}$; moreover, these representations have physical meaning, in that bosonic or fermionic (complex) quantum systems can very naturally be modelled in terms of the corresponding real or, respectively, quaternionic Hilbert spaces. Again, see, e.g., [4] a cogent development of this line of thought. In any case, it seems valuable to be able to delineate clearly what does and what does not depend on this assumption, particularly if we are interested in the possibilities for a "post-quantum" theory.

\footnotetext{
${ }^{3}$ And also notwithstanding my appeal to the Koecher-Vinberg Theorem, as this is itself a very accessible result. See [18] for a not terribly taxing proof. A number of the other reconstructions mentioned here also depend on nontrivial mathematical results, e.g., the classification of transitive actions of compact groups on spheres is used in [25].

${ }^{4}$ A real or quaternionic Hilbert space can be regarded as a complex Hilbert space equipped with a designated antiunitary operator $J$ satisfying, respectively, $J^{2}=1$ or $J^{2}=-1$; conversely, a complex Hilbert space is essentially equivalent to a real or quaternionic one equipped with, respectively, an orthogonal or a simplectic operator $J$ satisfying $J^{2}=-\mathbf{1}$ or $J^{2}=\mathbf{1}$.
} 
Organization The balance of this paper is arranged as follows. Section 2 provides general background on probabilistic models, ordered vector spaces, Jordan algebas and so on, making more precise many of the technical terms used above. This material will be familiar to many, but probably not to all, readers. Section 3 contains the proof of Theorem 1; Corollaries 1 and 2 are proved in Section 4. Section 5 collects some final thoughts, inlcuding a few further remarks on how the approach of this paper compares to the reconstructions of QM cited above. Appendix A contains additional information on probabilistic models associated with euclidean Jordan algebras, and Appendix B shows how a version of the "subspace axiom", plus a symmetry assumption, can replace some of the assumptions native to this paper.

Several of the ideas developed here were earlier explored, and somewhat similar results derived, in [30] and [31], but the approach taken here is much simpler and more direct, and seems to go a good deal farther.

\section{Background}

The mathematical framework for this paper is that of "generalized probabilistic theories" [10], in the idiom of $[30,9]$, which I now quickly review. ${ }^{5}$ In a few places, set off in numbered definitions, my usage differs slightly from that of these last-cited works. See $[18,2]$ for more information on ordered vector spaces and Jordan algebras.

Ordered vector spaces An ordered vector space is a real vector space $\boldsymbol{E}$ equipped with a closed, convex cone $\boldsymbol{E}_{+}$with $\boldsymbol{E}_{+} \cap-\boldsymbol{E}_{+}=\{0\}$ and $\boldsymbol{E}=\boldsymbol{E}_{+}-\boldsymbol{E}_{+}$- that is, $\boldsymbol{E}$ is spanned by $\boldsymbol{E}_{+}$. The cone induces a partial order, invariant under translation and multiplication by non-negative scalars, given by $a \leq b$ iff $b-a \in \boldsymbol{E}_{+}$. As an illustration, the space $\mathbb{R}^{X}$ of real-valued functions on a set $X$ is ordered by the cone $\mathbb{R}_{+}^{X}$ of functions taking non-negative values. Another example is the space $\mathcal{L}_{\text {sa }}(\mathcal{H})$ of hermitian operators on a real, complex or quaternionic Hilbert space, ordered by the cone of positive semi-definite operators.

A linear mapping $T: \boldsymbol{E} \rightarrow \boldsymbol{F}$ between ordered vector spaces is positive iff $T\left(\boldsymbol{E}_{+}\right) \subseteq \boldsymbol{F}_{+}$. If $T$ is bijective and $T^{-1}$ is also positive, then $T$ is an order isomorphism. If $\boldsymbol{E}$ and $\boldsymbol{F}$ are finite dimensional with $\operatorname{dim}(\boldsymbol{E})=\operatorname{dim}(\boldsymbol{F}), T$ is an order isomorphism iff $T\left(\boldsymbol{E}_{+}\right)=\boldsymbol{F}_{+}$. The dual space $\boldsymbol{E}^{*}$ of a finite-dimensional ordered linear space carries a natural ordering, defined by the dual cone, $\boldsymbol{E}_{+}^{*}$, consisting of positive linear functionals $f \in \boldsymbol{E}^{*}$.

Probabilistic Models As discussed above, a probabilistic model is characterized by a set $\mathcal{M}(A)$ of basic measurements or tests, and a set $\Omega(A)$ of states. It is convenient to identify each test with its outcome-set, so that $\mathcal{M}(A)$ is simply a collection of non-empty sets (a test space, in the language of [9]). Let $X(A)$ stand for the union of this collection; that is, $X(A)$ is the space of all outcomes of all basic measurements. States are understood as assignments of probabilities to measurement-outcomes, that is, as functions $\alpha: X(A) \rightarrow[0,1]$ such that $\sum_{x \in E} \alpha(x)=1$ for all tests $E \in \mathcal{M}(A)$ (but not all such functions necessarily correspond to states). As mentioned above, a state $\alpha \in \Omega(A)$ is non-singular iff $\alpha(x)>0$ for all $x \in X(A)$. To reflect the possibility of forming statistical mixtures, I also assume that $\Omega(A)$ is convex, that is, if $p_{1}, \ldots, p_{n}$ are non-negative real numbers summing to 1 , and $\alpha_{1}, \ldots, \alpha_{n}$ are states in $\Omega(A)$, then the function $p_{1} \alpha_{1}+\cdots+p_{n} \alpha_{n}$ also

\footnotetext{
${ }^{5}$ This is a variant of the standard "convex-operational" framework developed in the 1960s and 1970s by Ludwig, Davies and Lewis and others (e.g., $[24,15,16]$ ), specialized to finite dimensions, and with additional structure deriving from work of Foulis and Randall [17].
} 
belongs to $\Omega(A)$. Finally, I assume that $\Omega(A)$ is closed under pointwise limits, whence, compact as a subset of $[0,1]^{X(A)}$ in the product topology.

By way of illustration, in the simplest classical model, $\mathcal{M}(A)$ consists of a single, finite test, and $\Omega(A)$ is the simplex of all probability weights on that test. Of more immediate interest to us is the quantum model $A(\mathcal{H})=(\mathcal{M}(\mathcal{H}), \Omega(\mathcal{H}))$ associated with a complex Hilbert space $\mathcal{H}$. The test space $\mathcal{M}(\mathcal{H})$ is the set of orthonormal bases of $\mathcal{H}$; thus, the outcome-space $X(\mathcal{H})$ is the set of unit vectors of $\mathcal{H}$. The state space $\Omega(\mathcal{H})$ consists of the quadratic forms associated with density operators on $\mathcal{H}$, so that a state $\alpha \in \Omega(\mathcal{H})$ has the form $\alpha(x)=\left\langle W_{\alpha} x, x\right\rangle$ for some density operator $W_{\alpha}$, and all unit vectors $x \in X(\mathcal{H})$. Real and quaternionic quantum models, corresponding to real or quaternionic Hilbert spaces, are defined in the same way.

Remark: Not every physically accessible observable on a finite-dimensional quantum system is represented by an orthonormal basis. Rather, the general observable corresponds to a positiveoperator-valued measure. Similarly, for an arbitrary probabilistic model $A$, the test space $\mathcal{M}(A)$ may, but need not, represent a complete catalogue of all possible measurements one might make on the system represented by $A$ : rather, it is some privileged (or perhaps, simply convenient) catalogue of such measurements, sufficiently large to determine the system's states.

The spaces $\mathbf{V}(A)$ and $\mathbf{E}(A)$. Any probabilistic model $A$ gives rise in a canonical way to an ordered vector space $\mathbf{V}(A)$. This is simply the span of the state space $\Omega(A)$ in the space $\mathbb{R}^{X(A)}$, ordered by the cone $\mathbf{V}(A)_{+}$of non-negative multiples of states; that is, $\beta \in \mathbf{V}(A)_{+}$iff $\beta=t \alpha$ for some state $\alpha \in \Omega(A)$ and some real constant $t \geq 0$. An element of $\mathbf{V}(A)_{+}$of the form $t \alpha$ with $\alpha \in \Omega(A)$ and $t \leq 1$ is said to be sub-normalized. One can show that the interior of $\mathbf{V}(A)_{+}$ consists exactly of multiples of non-singular states. The dimension of a model $A$ is the dimension of $\mathbf{V}(A)$. As mentioned in the introduction, it is assumed in this paper that all probabilistic models are finite-dimensional.

There is a canonical positive linear functional $u_{A}: \mathbf{V}(A) \rightarrow \mathbb{R}$, called the unit effect of $A$, given by $u_{A}(\alpha)=\sum_{x \in E} \alpha(x)$, where $E$ is any test in $\mathcal{M}(A)$. Note that if $\alpha \in \mathbf{V}(A)_{+}$, then $u_{A}(\alpha)=1$ precisely when $\alpha \in \Omega(A)$, and that every non-zero element $\alpha \in \mathbf{V}(A)_{+}$has the form $\beta=t \alpha$ for a unique $t=u_{A}(\beta)>0$. Thus, any non-zero $\beta \in \mathbf{V}(A)_{+}$can be normalized to yield a state $\widetilde{\beta}=u_{A}(\beta)^{-1} \beta \in \Omega(A)$. A positive linear functional $f \in \mathbf{V}(A)_{+}^{*}$ with $f \leq u_{A}$ is usually called an effect on $A$, and can be thought of as representing an "in-principle" measurement outcome, with probability $0 \leq f(\alpha) \leq 1$ in state $\alpha$. Every outcome $x \in X(A)$ corresponds to an effect $\widehat{x}: \mathbf{V}(A) \rightarrow \mathbb{R}$, given by $\widehat{x}(\alpha)=\alpha(x)$ for all $\alpha \in \mathbf{V}(A)$, and $u_{A}=\sum_{x \in E} \widehat{x}$.

If $A=A(\mathcal{H})$ is the quantum mechanical model associated with a finite-dimensional Hilbert space $\mathcal{H}$, as discussed above, then, identifying $\Omega(\mathcal{H})$ with the convex set of density operators on $\mathcal{H}, \mathbf{V}(A)$ can be identified with the ordered vector space $\mathcal{L}(\mathcal{H})$ of self-adjoint operators on $\mathcal{H}$, with its usual cone of positive operators. We can then also identify $\mathbf{V}(\mathcal{H})^{*}$ with $\mathcal{L}(\mathcal{H})$, using the trace inner product. That is, if $a \in \mathcal{L}(\mathcal{H})$, we can define a positive linear functional $a \in \mathbf{V}(A)^{*}$ by setting $a(\alpha)=\operatorname{Tr}(a \alpha)$ for all $\alpha \in \mathbf{V}(A)=\mathcal{L}(\mathcal{H})$, and all such functionals arise uniquely from elements of $\mathcal{L}(\mathcal{H})$. In this setting, measurement-outcomes are unit vectors of $\mathcal{H}$, and, for each $x \in X(\mathcal{H})$, and for each density operator $\alpha$ on $\mathbf{V}(A), \widehat{x}(\alpha)=\operatorname{Tr}\left(\alpha p_{x}\right)$ where $p_{x}$ is the rank-one projection associated with $x$. More generally, effects correspond to positive operators between $\mathbf{0}$ and 1.

The spectral theorem for self-adjoint operators tells us that every effect in $\mathbf{V}(A(\mathcal{H}))=\mathcal{L}(\mathcal{H})$ is a positive linear combination of functionals $\widehat{x}$ corresponding to measurement outcomes. This will not be the case for probabilistic models in general. It is therefore useful to define a smaller cone, as follows: 
Definition 2. The space $\mathbf{E}(A)$ is the span, in $\mathbf{V}(A)^{*}$, of the set of effects $\widehat{x}$ associated with outcomes $x \in X(A)$, ordered by the cone $\mathbf{E}(A)_{+}$of finite linear combinations $\sum_{i} t_{i} \widehat{x}_{i}, x_{i} \in X(A)$, with coefficients $t_{i} \geq 0$.

Since we are assuming that $\mathbf{V}(A)$ and (hence) $\mathbf{V}(A)^{*}$ are finite-dimensional, the set of functionals $\widehat{x}$ in fact spans $\mathbf{V}(A)^{*}$ (since they separate points of $\mathbf{V}(A)$.) Thus, $\mathbf{E}(A)$ and $\mathbf{V}(A)^{*}$ are identical as vector spaces. However, their cones are generally quite different. If $a \in \mathbf{E}(A)_{+}$, then $a(\alpha) \geq 0$ for all $\alpha \in \mathbf{V}(A)_{+}$, so the cone $\mathbf{E}(A)_{+}$is contained in the dual cone $\mathbf{V}(A)_{+}^{*}$, but the inclusion is usually proper. Thus, $\mathbf{E}(A)$ and $\mathbf{V}(A)$ are generally distinct as ordered vector spaces.

Remark: The space $\mathbf{E}(A)$ will be a useful technical tool in what follows, but should not necessarily be regarded as anything more than that. In particular, it is not assumed that all physically meaningful effects reside in $\mathbf{E}(A)_{+}$, nor that every effect in $\mathbf{E}(A)_{+}$is physically meaningful. In fact, it will not be necessary to take any position at all on which effects, other than those associated with measurement outcomes, are physically significant. Thus, as mentioned in the introduction, we avoid the so-called no-restriction hypothesis [21], namely, the asumption that all effects in $\mathbf{V}(A)_{+}^{*}$ are physically accessible. This assumption is often made in the literature, sometimes explicitly (e.g., [25]), sometimes not.

Processes A physical process on a system represented by a probabilistic model $A$ is naturally represented by an affine (that is, convex-linear) mapping $T: \Omega(A) \rightarrow \mathbf{V}(A)$ such that, for every $\alpha \in \Omega(A), T(\alpha)=p \beta$ for some $\beta \in \Omega(A)$ and some constant $0 \leq p \leq 1$ (depending on $\alpha$ ), which we can regard as the probability that the process occurs, given that the initial state is $\alpha .{ }^{6}$ Such a mapping extends uniquely to a positive linear mapping $T: \mathbf{V}(A) \rightarrow \mathbf{V}(A)$ with $T(\alpha)\left(u_{A}\right) \leq 1$ for all $\alpha \in \Omega(A)$.

Definition 3. A process $T: \mathbf{V}(A) \rightarrow \mathbf{V}(A)$ is probabilistically reversible - hereafter, just $p$ reversible ${ }^{7}$ - iff there is another process, $S$, such that, for every state $\alpha$, there exists a constant $p \in(0,1]$ with $S(T(\alpha))=p \alpha$.

In other words, $S$ allows us to recover $\alpha$ from $T(\alpha)$, up to normalization. It is not hard to see that $p$ must be independent of $\alpha$, so that $S=p T^{-1}$. In particular, $T$ is an order-automorphism of $\mathbf{V}(A)$.

A process $T: \mathbf{V}(A) \rightarrow \mathbf{V}(B)$ has a dual action on $V(A)^{*}$, given by $T^{*}(f)=f \circ T$ for all $f \in \mathbf{V}(A)^{*}$, with $T^{*}\left(u_{A}\right) \leq u_{A}$. $T$ is lossless iff $T^{*}\left(u_{A}\right)=u_{A}$. In our finite-dimensional setting, we can identify $\mathbf{V}(A)^{*}$ with $\mathbf{E}(A)$ as vector spaces, but not, generally, as ordered vector spaces. While $T^{*}$ will preserve the dual cone $\mathbf{V}(A)_{+}^{*}$, it is not required, a priori, that $T^{*}$ preserve the cone $\mathbf{E}(A)_{+} \leq \mathbf{V}(A)^{*}$. This reflects the idea that not every physically accessible measurement need appear among the tests in $\mathcal{M}(A)$, as discussed above.

Self-Duality and Jordan Algebras. For both classical and quantum models, the ordered spaces $\mathbf{E}(A)$ and $\mathbf{V}(A)$ are isomorphic. In the former case, where $\mathcal{M}(A)$ consists of a single test $E$ and $\Omega(A)$ is the simplex of all probability weights on $E$, we have $\mathbf{V}(A) \simeq \mathbb{R}^{E}$ and $\mathbf{E}(A) \simeq\left(\mathbb{R}^{E}\right)^{*}$, with the standard inner product on $\mathbb{R}^{E}$ providing the order-isomorphism. If $\mathcal{H}$ is a finite-dimensional

\footnotetext{
${ }^{6}$ To be clear, we are not suggesting that all such positive mappings on $\mathbf{V}(A)$ represent physically allowable processes. Indeed, in QM, only completely positive mappings are physically allowable.

${ }^{7}$ It should be noted that my usage is slightly nonstandard here: ordinarily, the adjective reversible is reserved for processes that are probabilistically reversible, in the above sense, with probability one.
} 
real or complex Hilbert space, we have an affine isomorphism between the state space of $\Omega(\mathcal{H})$ and the set of density operators on $\mathcal{H}$, allowing us to identify $\mathbf{V}(A(\mathcal{H}))$ with the space $\mathcal{L}_{\text {sa }}(\mathcal{H})$ of self-adjoint operators on $\mathcal{H}$, ordered by the cone of positive operators. For any $x \in X(\mathcal{H})$, the evaluation functional $\widehat{x} \in \mathbf{V}(A)$ is then given by $W \mapsto\langle W x, x\rangle=\operatorname{Tr}\left(W P_{x}\right)$. It follows that $\mathbf{E}(A(\mathcal{H})) \simeq \mathcal{L}_{\mathrm{sa}}(\mathcal{H})^{*} \simeq \mathcal{L}_{\mathrm{sa}}(\mathcal{H})$, with the latter isomorphism implemented by the trace inner product.

More generally, call an inner product $\langle$,$\rangle on an ordered vector space \boldsymbol{E}$ positive iff $\langle a, b\rangle \geq 0$ for all $a, b \in \boldsymbol{E}_{+}$. We then have a positive linear mapping $\boldsymbol{E} \rightarrow \boldsymbol{E}^{*}$, namely $a \mapsto\langle a, \cdot\rangle$. If this is an order-isomorphism, one says that $\boldsymbol{E}$ is self-dual with respect to this inner product. This is equivalent to the condition $a \in \boldsymbol{E}_{+}$iff $\langle a, b\rangle \geq 0$ for all $b \in \boldsymbol{E}_{+}$. In this language, the standard inner product on $\mathbb{R}^{E}$ and the trace inner product on $\mathcal{L}_{\mathrm{sa}}(\mathcal{H})$ are self-dualizing, for any finite set $E$ and finite-dimensional Hilbert space $\mathcal{H}$.

In fact, any euclidean Jordan algebra, ordered by its cone of squares, is self-dual with respect to its canonical inner product. Recall here that a Jordan algebra is a real commutative (but not necessarily associative) unital algebra $(\boldsymbol{J}, \bullet)$ satisfying the Jordan identity

$$
a \cdot\left(a^{2} \cdot b\right)=a^{2} \cdot(a \cdot b)
$$

for all $a, b \in \boldsymbol{J}$ (with $a^{2}=a \bullet a$ ). A euclidean Jordan algebra (EJA) is a finite-dimensional Jordan algebra $\boldsymbol{J}$ equipped with an inner product $\langle$, $\rangle$ such that $\langle a \bullet b, c\rangle=\langle b, a \bullet c\rangle$ for all $a, b, c \in \boldsymbol{J}$. This is equivalent to the condition that $\boldsymbol{J}$ be formally real, i.e, that $\sum_{i=1}^{k} a_{i}^{2}=0$ implies $a_{i}=0$ for all $i$. A EJA $\boldsymbol{J}$ is also an ordered vector space with positive cone $\boldsymbol{J}_{+}=\left\{a^{2} \mid a \in \mathbf{E}\right\}$, and it can be shown that this cone is self-dual with respect to the given inner product [18]. Examples of euclidean Jordan algebras include the space $\mathcal{L}_{\mathrm{sa}}(\mathcal{H})$ of self-adjoint operators on a finite-dimensional real, complex or quaternionic Hilbert space $\mathcal{H}$, with $a \cdot b=\frac{1}{2}(a b+b a)$, and with $\langle a \mid b\rangle=\operatorname{Tr}(a b)$. The exceptional Jordan algebra of self-adjoint hermitian matrices over the Octonions is also a euclidean Jordan algebra. Finally, one obtains a euclidean Jordan algebra, called a spin factor, by defining on $V_{n}:=\mathbb{R} \times \mathbb{R}^{n}$ a product $(t, \mathbf{x}) \cdot(s, \mathbf{y})=(t s+\langle\mathbf{x}, \mathbf{y}\rangle, t \mathbf{y}+s \mathbf{x})$. This essentially exhausts the possibilities: according to the Jordan-von Neumann-Wigner classification theorem [22], every euclidean Jordan algebra is a direct sum of euclidean Jordan algebras of these five types.

We can associate a probabilistic model to an EJA $\boldsymbol{J}$ in the following way. An idempotent in $\boldsymbol{J}$ is an element $e=e^{2}$. An idempotent is minimal, or primitive, iff for any idempotent $f \leq e$, $f=0$ or $f=e$. Two idempotents $e, f$ are Jordan-orthogonal iff $e \cdot f=0$. A maximal pairwise Jordan-orthogonal set $\left\{e_{1}, \ldots, e_{n}\right\}$ of primitive idempotents summing to the Jordan unit is called a Jordan frame.

Definition 4. The Jordan model $A(\boldsymbol{J})=(X(\boldsymbol{J}), \mathcal{M}(\boldsymbol{J}), \Omega(\boldsymbol{J}))$ corresponding to a euclidean Jordan algebra $\boldsymbol{J}$ has $X(\boldsymbol{J})$ the set of primitive idempotents, $\mathcal{M}(\boldsymbol{J})$, the set of Jordan frames of $\boldsymbol{J}$, and $\Omega(\boldsymbol{J})$ the set of states of the form $\alpha(x):=\langle a, x\rangle$ where $a \in \mathbf{E}(A)_{+}$satisfies $\langle a, \mathbf{1}\rangle=1$. The spectral theorem for EJAs ([18], Theorem III.1.2) expresses every $a \in \boldsymbol{J}$ in the form $a=\sum_{x \in E} t_{x} x$ where $E$ is a Jordan frame. Therefore, $\boldsymbol{J}=\mathbf{E}(A)$, and the model $A(\boldsymbol{J})$ is self-dual.

Besides self-duality, all euclidean Jordan algebras share a property called homogeneity: the group of order-automorphisms of $\boldsymbol{J}$ acts transitively on the interior of the positive cone $\boldsymbol{J}_{+}$. The Koecher-Vinberg Theorem [18] states that, conversely, any finite-dimensional homogeneous, self-dual, homogenous ordered vector space $\boldsymbol{J}$ can be equipped with the structure of a euclidean Jordan algebra.

Definition 5. A probabilistic model $A$ is homogeneous iff $\mathbf{V}(A)$ is homogeneous, and self-dual iff $\mathbf{E}(A)$ carries an inner product with respect to which it is self-dual and $\mathbf{E}(A)_{+} \simeq \mathbf{V}(A)_{+}$, in the 
sense that $a \in \mathbf{E}(A)_{+}$iff $\alpha(x):=\langle a, \widehat{x}\rangle$ defines an element of $\mathbf{V}(A)_{+}$, and every element of $\mathbf{V}(A)_{+}$ arises in this way.

If $A$ is both homogeneous and self-dual - henceforth, HSD - then $\mathbf{E}(A)$ is also homogeneous and self-dual, and thus, by the Koecher-Vinberg Theorem, can be made into a Jordan algebra. In Appendix $\mathrm{A}$, it is shown that this can be done in such a way that $A$ is actually isomorphic to the Jordan model corresponding to $\mathbf{E}(A)$.

Bipartite States and Conditioning A joint probability weight on a pair of models $A$ and $B$ is a mapping $\omega: X(A) \times X(B) \rightarrow \mathbb{R}$ such that, for all $E \in \mathcal{M}(A)$ and $F \in \mathcal{M}(B)$,

$$
\sum_{(x, y) \in E \times F} \omega(x, y)=1 .
$$

Such a weight is said to be non-signaling if, in addition, the marginal weights

$$
\omega_{1}(x):=\sum_{y \in F} \omega(x, y) \text { and } \omega_{2}(y):=\sum_{x \in E} \omega(x, y)
$$

are well-defined, i.e., independent of the choice of tests $F \in \mathcal{M}(B)$ and $E \in \mathcal{M}(A)$, respectively. The idea is that such a state precludes the sending of signals between $A$ and $B$ based solely on the choice of what test to perform.

If $\omega$ is non-signalling, then given outcomes $y \in X(B)$ and $x \in X(A)$, we can define conditional probability weights $\omega_{1 \mid y}$ and $\omega_{2 \mid x}$ on $A$ and $B$, respectively, by setting

$$
\omega_{1 \mid y}(x)=\frac{\omega(x, y)}{\omega_{2}(y)} \text { and } \omega_{2 \mid x}(y):=\frac{\omega(x, y)}{\omega_{1}(x)},
$$

when $\omega_{2}(y)$ and $\omega_{1}(x)$ are non-zero. This gives us the following bipartite law of total probability $[17]$

$$
\omega_{2}=\sum_{x \in E} \omega_{1}(x) \omega_{2 \mid x} \text { and } \omega_{1}=\sum_{y \in F} \omega_{2}(y) \omega_{1 \mid y}
$$

which will be exploited below.

Definition 6. Let $\omega$ be a non-signaling joint probability weight on $A$ and $B$. If all conditional weights $\omega_{1 \mid x}$ and $\omega_{2 \mid y}$ (and hence, the marginals $\omega_{1}$ and $\omega_{2}$ ) of $\omega$ belong to $\Omega(A)$ and $\Omega(B)$, respectively, then we say that $\omega$ is a bipartite state on the models $A$ and $B$.

If $\mathcal{H}$ and $\mathcal{K}$ are real or complex Hilbert spaces, every density operator $W$ on $\mathcal{H} \otimes \mathcal{K}$ gives rise to a bipartite state on $A(\mathcal{H})$ and $A(\mathcal{K})$, given by $\omega(x, y)=\langle W x \otimes y, x \otimes y\rangle$.

The conditioning map If $\omega$ is a bipartite state on $A$ and $B$, define the associated conditioning maps $\widehat{\omega}: X(A) \rightarrow \mathbf{V}(B)$ and $\widehat{\omega}^{*}: X(B) \rightarrow \mathbf{V}(A)$ by

$$
\widehat{\omega}(x)(y)=\omega(x, y)=\widehat{\omega}^{*}(y)(x) .
$$

Note that $\widehat{\omega}(x)=\omega_{1}(x) \omega_{2 \mid x}$ for every $x \in X(A)$, i.e., $\widehat{\omega}(x)$ can be understood as the un-normalized conditional state of $B$ given the outcome $x$ on $A$, and similarly for $\widehat{\omega}^{*}(y)$.

The conditioning map $\widehat{\omega}$ extends uniquely to a positive linear mapping $\mathbf{E}(A) \rightarrow \mathbf{V}(B)$, which I also denote by $\widehat{\omega}$, such that $\widehat{\omega}(\widehat{x})=\widehat{\omega}(x)$ for all outcomes $x \in X(A)$. (To see this, consider the positive linear mapping $T: \mathbf{V}(A)^{*} \rightarrow \mathbb{R}^{X(B)}$ defined, for $f \in \mathbf{V}(A)^{*}$, by $T(f)(y)=f\left(\widehat{\omega}^{*}(y)\right)$ 
for all $y \in X(B)$. If $f=\widehat{x}$, we have $T(\widehat{x})=\omega_{1}(x) \omega_{2 \mid x} \in \mathbf{V}(B)_{+}$. Thus, the range of $T$ lies in $\mathbf{V}(B)$.) In the same way, $\widehat{\omega}^{*}$ defines a positive linear mapping $\widehat{\omega}^{*}: \mathbf{E}(B) \rightarrow \mathbf{V}(A)$. Notice that $\widehat{\omega}$ need not take $\mathbf{V}(A)_{+}^{*}$ into $\mathbf{V}(B)_{+}$. This is the principal reason for working with $\mathbf{E}(A)$ rather than $\mathbf{V}(A)^{*}$. If $\widehat{\omega}: \mathbf{E}(A) \rightarrow \mathbf{V}(A)$ is an order isomorphism, $\omega$ is said to be an isomorphism state [5].

Composite Systems As the language here suggests, one wants to view (some) bipartite states as elements of the state space of a composite model. Broadly, a composite of two probabilistic models $A$ and $B$, is a model $A B$ equipped with a mapping $X(A) \times X(B) \rightarrow \mathbf{V}(A B)_{+}^{*}$, taking every pair of outcomes $x \in X(A)$ and $y \in X(B)$, to a product effect $x y$, such that every state $\omega \in \Omega(A B)$ pulls back to a bipartite state $\omega(x, y):=\omega(x y)$ on $A$ and $B$. While nothing in the mathematical development to follow depends on the choice of such a composite model, questions of interpretation may hinge on such a choice.

\section{Conjugate Systems}

Let $\mathcal{H}$ be an $n$-dimensional complex Hilbert space and $\overline{\mathcal{H}}$ is its conjugate space. As discussed in the introduction, the maximally engangled "EPR" state, defined by $\Psi=\frac{1}{\sqrt{n}} \sum_{x \in E} x \otimes \bar{x}$, where $E$ is any orthonormal basis for $\mathcal{H}$, establishes a perfect, uniform correlation betweeen every projection-valued observable on the system associated with $\mathcal{H}$, and its counterpart on $\overline{\mathcal{H}}$. Moreover, $\Psi$ effectively defines the normalized trace inner product on $\mathbf{E}(\mathcal{H})=\mathcal{L}_{\text {sa }}(\mathcal{H})$. Since it is precisely this inner product that makes $\mathcal{L}_{\text {sa }}(\mathcal{H})$ self-dual, one might guess that the existence of a uniformly correlating bipartite state is implicated in self-duality more generally.

As a first step, we need to generalize the relationship between the models $A(\mathcal{H})$ and $A(\overline{\mathcal{H}})$. In order to do this, as mentioned in the introduction, we need first to impose the minor restriction that, henceforth, all models are uniform, meaning that all tests have a common cardinality $n$, and that the maximally mixed state $\rho$, given by $\rho(x)=1 / n$ for all $x \in X(A)$, belongs to $\Omega(A)$. An isomorphism between two models $A$ and $B$ is a bijection $\phi: X(A) \rightarrow X(B)$ such that $\phi(E) \in \mathcal{M}(B)$ iff $E \in \mathcal{M}(A)$, and $\beta \circ \phi \in \Omega(A)$ iff $\beta \in \Omega(B)$. It is straightforward that such a mapping gives rise to an order isomorphism - which I'll also denote by $\phi$ - from $\mathbf{E}(A)$ to $\mathbf{E}(B)$, defined by $\phi(\widehat{x})=\widehat{\phi(x)}$ for all $x \in X(A)$. The following reprises, and makes more precise, the definition of a conjugate system (Definition 1 ).

Definition 1 (bis) Let $A$ be a uniform probabilistic model of rank $n$. A conjugate for $A$ is a triple $\left(\bar{A}, \gamma_{A}, \eta_{A}\right)$ consisting of a probabilistic model $\bar{A}$, an isomorphism $\gamma_{A}: A \simeq \bar{A}$, and a bipartite state $\eta_{A}$ on $A$ and $\bar{A}$ such that

(a) $\eta_{A}\left(x, \gamma_{A}(x)\right)=1 / n$ for every $x \in X(A)$.

(b) Every state $\alpha \in \Omega(A)$ is the marginal of some bipartite state $\omega$ on $A$ and $\bar{A}$ that correlates some test $E \in \mathcal{M}(A)$ with its counterpart on $\bar{A}$, so that $\omega(x, \gamma(x))=\alpha(x)$ for every $x \in E$.

As remarked earlier, the marginals of the perfectly correlating state $\eta$ in part (a) are the maximally mixed states $\rho$ on $A$ and $\bar{\rho}$ on $A$ and $\bar{A}$, respectively. Where no ambiguity is likely, I write $\bar{x}$ for $\gamma_{A}(x)$. If $\bar{A}$ satisfies (a), but not necessarily (b), I will call it a weak conjugate for $A$.

Given any bipartite state on $A$ and $\bar{A}$ satisfying (a) i.e., $\eta\left(x, \gamma_{A}(x)\right)=1 / n$ for all $x \in X(A)$, the bipartite state $\eta^{t}$ defined by $\eta^{t}(x, \gamma(y))=\eta(y, \gamma(x))$ is also perfectly uniformly correlating, 
whence, so is the symmetic state $\left(\eta+\eta^{t}\right) / 2$. We therefore can, and do, assume in what follows that the chosen correlating state $\eta_{A}$ is always symmetric. If $A$ is sharp, it is easy to show that $\eta$ is uniquely determined by condition (a) of Definition 1: since $\eta(x, \bar{y})=0$ for outcomes $y \neq x$ belonging to a common test, and $\eta(y, \bar{y})=1 / n$, we have $\eta_{1 \mid \bar{y}}(y)=1$, i.e,.. $\eta_{1 \mid \bar{y}}=\delta_{y}$ where $\delta_{y}$ is the unique state in which $y$ has probability 1 . Thus, $\eta(x, \bar{y})=\delta_{y}(x)$. In this case, therefore, $\eta=\eta^{t}$, i.e., $\eta$ is automatically symmetric. ${ }^{8}$

If $A(\mathcal{H})$ is the quantum probabilistic model associated with a finite-dimensional Hilbert space, then the EPR state $\Psi$ turns $\bar{A}(\mathcal{H}):=A(\overline{\mathcal{H}})$ into a conjugate in this sense, with $\eta_{A}(x, \bar{y})=$ $|\langle\Psi, x \otimes \bar{y}\rangle|^{2}$. In fact, as pointed out in the introduction, $A(\overline{\mathcal{H}})$ is a conjugate for $A(\mathcal{H})$, since every density operator is the marginal of a state $\Psi_{W}$ correlating an eigenbasis for $W$ with its conjugate. All of this works equally well for real quantum systems, taking $\overline{\mathcal{H}}=\mathcal{H}$. With a little care, it can be shown to work for quaternionic systems as well.

Remark: One might wonder whether one can use the isomorphism $\gamma_{A}$ to identify $A$ with its conjugate. Certainly one can define a bipartite state $\eta_{A}^{\prime}(x, y)=\eta_{A}\left(x, \gamma_{A}(y)\right)$. However, whether this corresponds to a legitimate state of any physically reasonable composite $A A$ of $A$ with itself, depends on the particular probabilistic theory at hand. For example, if $A=A(\mathcal{H})$ is the quantum model associated with a complex Hilbert space $\mathcal{H}$, then $(a, b) \mapsto \operatorname{Tr}(a b)$ corresponds to no state on $A(\mathcal{H} \otimes \mathcal{H})$. On the other hand, any choice of an anti-unitary operator $J$ acting on $\mathcal{H}$ yields a unitary isomorphism $\bar{J}: \mathcal{H} \rightarrow \overline{\mathcal{H}}$, given by $\bar{J}(x)=\overline{J(x)}$ for all $x \in \mathcal{H}$. Defining $\eta^{\prime}(x, y):=\left|\left\langle(\mathbf{1} \otimes \bar{J})^{-1} \Psi, x \otimes y\right\rangle\right|^{2}=|\langle\Psi, x \otimes \bar{J} y\rangle|^{2}$ gives a state on $A(\mathcal{H} \otimes \mathcal{H})$, correlating along the anti-unitary isomorphism $\gamma^{\prime}(x):=J^{-1} x$. Thus, whether we choose to treat $A(\mathcal{H})$ as its own conjugate, or as distinct from its conjugate, is to an extent a matter of convention.

Conjugates and Self-Duality We are now ready to prove Theorem 1, which, for convenience, I restate. Recall that a model $A$ is sharp iff, for every outcome $x \in X(A)$, there is a unique state $\delta_{x} \in \Omega(A)$ with $\delta_{x}(x)=1$. Both classical and quantum models are sharp.

Theorem 1 (bis) Let $A$ be sharp and have a conjugate $\bar{A}$. Then $\langle a, b\rangle:=\eta_{A}\left(a, \gamma_{A}(b)\right)$ is a self-dualizing inner product on $\mathbf{E}(A)$, and induces an order-isomorphism $\mathbf{E}(A) \rightarrow \mathbf{V}(A)$ given by $a \mapsto \eta_{A}(a, \cdot)$ for $a \in \mathbf{E}(A) .{ }^{9}$

The proof is not difficult. It will be convenient to break it up into a sequence of even easier lemmas. In the interest of readability, below I conflate $x \in X(A)$ with the corresponding effect $\widehat{x} \in \mathbf{E}(A)$, and write $\bar{x}$ for $\gamma_{A}(x)$. Until further notice, the hypotheses of Theorem 1 are in force. The first step is to obtain a kind of weak "spectral" decomposition for states in $\Omega(A)$ in terms of the states $\delta_{x}$.

Lemma 1. For every $\alpha \in \mathbf{V}(A)_{+}$, there exists a test $E$ such that

$$
\alpha=\sum_{x \in E} \alpha(x) \delta_{x}
$$

Proof: We can assume that $\alpha$ is a normalized state, i.e, that $\alpha \in \Omega(A)$. Since $\bar{A}$ is a conjugate for $A, \alpha=\omega_{1}$ where $\omega$ correlates some test $E \in \mathcal{M}(A)$ with $\bar{E} \in \mathcal{M}(\bar{A})$ along the bijection

\footnotetext{
${ }^{8}$ It follows that, where $\delta_{x}$ and $\delta_{y}$ are the unique states making $x$ and $y$ certain, we have $\delta_{y}(x)=\delta_{x}(y)$ for all $x, y \in X(A)$. Indeed, this last condition could substitute for condition (a) in the definition of $\eta$, as it implies that $\eta(x, \bar{y}):=\delta_{x}(y)$ is a valid non-signaling probabilty weight on $A$ and $\bar{A}$.

${ }^{9}$ Here, I am identifying $\mathbf{V}(A)$, as a vector space, with $\mathbf{E}(A)$.
} 
$x \mapsto \bar{x}$. By the law of total probability (1) for non-signaling states, $\alpha=\sum_{\bar{x} \in \bar{E}} \omega_{2}(\bar{x}) \omega_{1 \mid \bar{x}}$. Since $\omega$ is correlating we have $\omega_{1 \mid \bar{x}}(x)=1$. Thus, by sharpness, $\omega_{1 \mid \bar{x}}=\delta_{x}$. Hence, $\alpha=\sum_{x \in E} \omega_{2}(\bar{x}) \delta_{x}$. It follows that $\omega_{2}(\bar{x})=\alpha(x)$ for every $x \in E$, giving us (4).

We will refer to the decomposition in equation (4) as a spectral decomposition for $\alpha$.

Lemma 2. $\eta_{A}$ is an isomorphism state.

Proof: We need to show that $\widehat{\eta_{A}}: \mathbf{E}(A) \rightarrow \mathbf{V}(A)$ is an order-isomorphism. Since $\mathbf{E}(A)$ and $\mathbf{V}(A)$ have the same dimension, it is enough to show that $\widehat{\eta_{A}}$ maps the positive cone of $\mathbf{E}(A)$ onto that of $\mathbf{V}(\bar{A})$. Since $x \mapsto \bar{x}$ is an isomorphism between $A$ and $\bar{A}$, we can apply Lemma 1 to $\bar{A}$ : if $\alpha \in \mathbf{V}_{+}(\bar{A})$, we have $\alpha=\sum_{x \in E} \alpha(\bar{x}) \delta_{\bar{x}}$. Since $\eta_{A}(x, \bar{x})=1 / n$, we have $\widehat{\eta_{A}}(x)=\frac{1}{n} \delta_{\bar{x}}$ for every $x \in X(A)$. Hence, $\widehat{\eta_{A}}\left(\sum_{x \in E} n \alpha(x) x\right)=\alpha$.

Lemma 3. Every $a \in \mathbf{E}(A)$ has a representation $a=\sum_{x \in E} t_{x} x$ for some test $E \in \mathcal{M}(A)$ and some coefficients $t_{x}$.

Proof: If $a \in \mathbf{E}(A)_{+}$, then by Lemma $1, \widehat{\eta_{A}}(a)=\sum_{x \in E} t_{x} \delta_{\bar{x}}$ for some $E \in \mathcal{M}(A)$ and coefficients $t_{x} \geq 0$. By Lemma $2, \widehat{\eta}_{A}$ is an order-isomorphism. Applying $\widehat{\eta}_{A}^{-1}$ to the expansion above gives $a=\sum_{x \in E} t_{x} x$. For an arbitrary $a \in \mathbf{E}(A)$, we have $a=a_{1}-a_{2}$ with $a_{1}, a_{2} \in \mathbf{E}(A)_{+}$. Choose $N \geq 0$ with $a_{2} \leq N u$. Thus, $b:=a+N u_{A}=a_{1}+\left(N u_{A}-a_{2}\right) \geq 0$. So $b:=\sum_{x \in E} t_{x} x$ for some $E \in \mathcal{M}(A)$ and thus

$$
a=b-N u_{A}=\sum_{x \in E} t_{x} x-N\left(\sum_{x \in E} x\right)=\sum_{x \in E}\left(t_{x}-N\right) x .
$$

Lemma 4. The function $\langle a, b\rangle:=\eta_{A}\left(a, \gamma_{A}(b)\right)$ is an inner product on $\mathbf{E}(A)$.

Proof: $\eta_{A}$ is bilinear and, by assumption, symmetric. We need to show that $\langle$,$\rangle is positive$ definite. Let $a \in \mathbf{E}(A)$. From Lemma 3, we have $a=\sum_{x \in E} t_{x} x$ for some test $E$ and coeffcients $t_{x}$. Now

$$
\langle a, a\rangle=\left\langle\sum_{x \in E} t_{x} x, \sum_{y \in E} t_{y} y\right\rangle=\sum_{x, y \in E \times E} t_{x} t_{y}\langle x, y\rangle=\sum_{x, y \in E \times E} t_{x} t_{y} \eta_{A}(x, \bar{y})=\frac{1}{n} \sum_{x \in E} t_{x}^{2} \geq 0 .
$$

This is zero only when all coefficients $t_{x}$ are zero, i.e., only for $a=0$.

Proof of Theorem 1, concluded: Lemma 2 tells us that $\mathbf{E}(A) \simeq \mathbf{V}(A)$, so it remains only to show that the inner product $\langle$,$\rangle is self-dualizing. Clearly \langle a, b\rangle=\eta(a, \bar{b}) \geq 0$ for all $a, b \in \mathbf{E}(A)_{+}$. Suppose $a \in \mathbf{E}(A)$ is such that $\langle a, b\rangle \geq 0$ for all $b \in \mathbf{E}(A)_{+}$. Then $\langle a, y\rangle \geq 0$ for all $y \in X$. By Lemma $3, a=\sum_{x \in E} t_{x} x$ for some test $E$. Thus, for all $y \in E$ we have $\langle a, y\rangle=t_{y} \geq 0$, whence, $a \in \mathbf{E}(A)_{+}$.

Remarks There are several directions in which we can usefully modify the assumptions of Theorem 1 .

(1) In the proof of Theorem 1 , the only point at which we needed to assume that $\bar{A}$ satisfies condition (b) in the definition of a conjugate was in order to obtain the spectral decomposition - equation (4) - of Lemma 1. Thus, if we are willing simply to assume such decompositions are available, as in [7], then a weak conjugate suffices. Alternatively, any postulate or postulates leading to such decompositions can replace condition (b). For instance, certain versions of the symmetry and "subspace" axioms used in [20, 14, 25] imply a spectral decomposition. This is 
spelled out in Appendix B. Another approach to obtaining such a decomposition can be found in a recent paper of G. Chiribella and C. M. Scandolo [12].

(2) In fact, it is even enough if (4) holds for states in the interior of $\Omega(A)$. From this we have, as in the proof of Lemma 2, that the interior, $\mathbf{V}_{+}^{\circ}$, of the cone $\mathbf{V}(A)_{+}$is contained in $\widehat{\eta}_{A}\left(\mathbf{E}_{+}\right)$, from which it follows that $\widehat{\eta}$ is a linear isomorphism, and hence (as the vector spaces involved are finite-dimensional) an homeomorphism. Thus, $\widehat{\eta}_{A}\left(\mathbf{E}_{+}\right)$is closed, and so, contains the closure of $\mathbf{V}_{+}^{\circ}$, i.e., $\mathbf{V}_{+}$. In other words, $\hat{\eta}$ is an order-isomorphism. The proofs of Lemmas 3 and 4 , and the rest of the proof of Theorem 1, then proceed just as before.

(3) The definition of a conjugate for a probabilistic model $A$ requires the existence of the uniformly, universally correlating state $\eta_{A}$, and that arbitrary states of $A$ arise as marginals of bipartite states on $A$ and $\bar{A}$ correlating some test $E \in \mathcal{M}(A)$ with its conjugate twin. One might wonder whether there is some reasonably simple postulate that will imply both of these conditions. Suppose that $G$ is a group acting transitively on the outcome-space $X(A)$ of the model $A$, and leaving the state-space $\Omega(A)$ invariant. If $G$ is compact, there will exist an invariant state, $\rho$, obtained by group averaging; by the transitivity of $G$ on outcomes, this state must be constant. It follows that all tests have the same finite size size, say $n$, and that $\rho$ is the maximally mixed state $\rho(x) \equiv 1 / n$. That is, the model is uniform. Now let $\gamma_{A}: x \mapsto \bar{x}$ be an isomorphism between $A$ and a model $\bar{A}$. Suppose that every state $\alpha \in \Omega(A)$ is the marginal of a correlating state $\omega \in \Omega(A \bar{A})$ such that $\omega(g x, \overline{g y})=\omega(x, y)$ for all $g \in G$ with $\alpha \circ g=\alpha$. It is easily checked that this is satisfied by finite-dimensional quantum models. Applied to the maximally mixed state $\rho$, this produces a perfectly, uniformly correlating state $\eta_{A}$. Thus, $\bar{A}$ is a conjugate in the sense of Definition 3.

\section{Filters}

We have just seen that if $A$ is sharp and has a conjugate, then $\mathbf{E}(A)$ is self-dual, and isomorphic to $\mathbf{V}(A)$. Suppose now that every non-singular state of $A$ can be prepared, up to normalization, from the maximally mixed state $\rho(x) \equiv 1 / n$ by some reversible process. This guarantees that $\mathbf{V}(A)$, and hence, $\mathbf{E}(A)$, is homogeneous, so that, by the Koecher-Vinberg Theorem, $\mathbf{E}(A)$ carries a euclidean Jordan structure making $\mathbf{E}(A)_{+}$the cone of squares.

In fact, we can say something more interesting. In many kinds of laboratory experiments, the distinct outcomes of an experiment correspond to physical detectors, the efficiency of which can independently be attenuated, if desired, by the experimenter. This can always be done through post-processing, using a classical filter. In QM, it can also be accomplished by subjecting the system to a suitable process prior to measurement. To see this, let $A$ be a finite-dimensional quantum system, with corresponding Hilbert space $\mathcal{H}$, and identify $\mathbf{E}(A)$ with $\mathcal{L}_{\text {sa }}\left(\mathcal{H}_{A}\right)$. If $E$ is an orthonormal basis representing a basic measurement on this system, define a positive operator $V: \mathcal{H} \rightarrow \mathcal{H}$ by setting $V x=t_{x}^{1 / 2} x$ for every $x \in E$, where $0 \leq t_{x} \leq 1$. This gives us a completely positive linear mapping $\Phi: \mathbf{E}(A) \rightarrow \mathbf{E}(A)$, namely $\Phi(a)=V a V$. If $t_{x}>0$ for every $x \in E, \Phi$ has a completely positive inverse $\Phi^{-1}(a)=V^{-1} a V^{-1}$. For each $x \in E$, the corresponding effect $\widehat{x} \in \mathbf{E}(A) \simeq \mathcal{L}_{\mathrm{sa}}(\mathcal{H})$ is the rank-one projection operator $p_{x}$. It is easy to check that $V p_{x} V=t_{x} p_{x}$, i.e., that $\Phi(\widehat{x})=t_{x} \widehat{x}$ for every $x \in E$.

Definition 7. A filter for a test $E$ of a probabilistic model $A$ is a positive linear mapping $\Phi: \mathbf{V}(A) \rightarrow \mathbf{V}(A)$ such that, for every outcome $x \in E$, there exists a coefficient $0 \leq t_{x} \leq 1$ with

$$
\Phi(\alpha)(x)=t_{x} \alpha(x)
$$

for all all states $\alpha \in \Omega(A)$. Equivalently, $\Phi^{*}(x)=t_{x} x$ for every $x \in E$. 
As noted above, in QM, not only do filters with arbitrary coefficients exist for every test, but they can be implemented $p$-reversibly, so long as the coefficients $t_{x}$ are all non-zero. I will say that a general probabilistic model with this feature has arbitrary reversible filters.

Corollary 1 (bis) Suppose that $A$ is sharp and has a conjugate $\bar{A}$. If $A$ has arbitrary reversible filters, then $\mathbf{E}(A)$ is homogeneous and self-dual.

Proof: $A$ is self-dual by Theorem 1. Let $\alpha$ be a normalized state in the interior of $\mathbf{V}(A)_{+}$. By Lemma 1, $\alpha$ has a spectral decomposition $\alpha=\sum_{x \in E} \alpha(x) \delta_{x}$. Let $\Phi$ be a filter for $E$ with coefficients $\alpha(x)$. Since $\alpha$ is non-singular, $\alpha(x)>0$ for all $x \in E$, so $\Phi$ can be chosen to be reversible. Now expand the maximally mixed state $\rho$, with $\rho(x) \equiv 1 / n$, as $\rho=\sum_{x \in E} \frac{1}{n} \delta_{x}$. Then $\Phi(\rho)=\frac{1}{n} \sum_{x \in E} \alpha(x) \delta_{x}=\frac{1}{n} \alpha$. Thus, any non-singular state can be prepared, up to normalization, by a reversible filter, and it follows that $\mathbf{V}(A)$ is homogeneous. In view of Theorem $1, \mathbf{E}(A)$ is self-dual, and $\mathbf{E}(A) \simeq \mathbf{V}(A)$, whence, also homogeneous.

State preparation by reversible filters Suppose now that $A$ has only a weak conjugate $\bar{A}$, and that $\Phi$ is a filter for a test $E \in \mathcal{M}(A)$. By applying $\Phi$ to one of the two systems $A$ and $\bar{A}$, we can convert the correlator $\eta_{A}$ into a new sub-normalized bipartite state $\omega$, given by $\omega(x, y)=$ $\eta_{A}\left(\Phi^{*} x, y\right)$ for all $x \in X(A), y \in X(B)$. Noticing that $\Phi^{*}(x)=t_{x} x$ for every $x \in E$, we see that $\omega$ correlates $E$ with $\bar{E}$ : if $x, y \in E$ with $x \neq y$, we have

$$
\omega(x, \bar{y})=\eta_{A}\left(t_{x} x, \bar{y}\right)=t_{x} \eta_{A}(x, \bar{y})=0 .
$$

In other words, $\omega$ is correlating. It follows that the normalized bipartite state

$$
\widetilde{\omega}:=\frac{1}{\omega\left(u_{A}, u_{\bar{A}}\right)} \omega
$$

is likewise correlating. Since $\omega_{1}=\Phi(\rho)$, it follows that any state preparable from $\rho$ by a filter that is, any state of the form $\alpha=\widehat{\Phi(\rho)}$, where $\Phi$ is a filter and $\widetilde{\alpha}:=\frac{1}{u_{A}(\alpha)} \alpha-$ is the marginal of a correlating state, and hence enjoys a spectral decomposition as in Equation (4). Thus, if every state is so preparable, the weak conjugate $\bar{A}$ is actually a conjugate. So, in the presence of sharpness, we can replace the assumption that the conjugate is strong, by the requirement that every state be preparable by a filter. In fact, by strengthening this preparability assumption, it is even possible to omit the hypothesis that $A$ is sharp.

The isomorphism $\gamma_{A}: A \simeq \bar{A}$ extends to an order-automorphism $\mathbf{V}(A) \simeq \mathbf{V}(\bar{A})$, given by $\alpha \mapsto \bar{\alpha}$, with $\bar{\alpha}(\bar{x})=\alpha(x)$ for all $x \in X(A)$. Hence, a positive linear mapping $\Phi: \mathbf{V}(A) \rightarrow \mathbf{V}(A)$ has a counterpart $\bar{\Phi}: \mathbf{V}(\bar{A}) \rightarrow \mathbf{V}(\bar{A})$, given by $\bar{\Phi}(\bar{\alpha})=\overline{\Phi(\alpha)}$. Let us say that $\Phi$ is symmetric with respect to $\eta_{A}$ iff $\eta_{A}\left(\Phi^{*}(x), \bar{y}\right)=\eta_{A}\left(x, \overline{\Phi^{*}}(y)\right)$ for all $x, y \in X(A)$, i.e., iff $\eta_{A} \circ\left(\Phi^{*} \otimes \mathrm{id}_{\bar{A}}\right)=$ $\eta_{A} \circ\left(\operatorname{id}_{A} \otimes \bar{\Phi}^{*}\right)$.

Lemma 5 Let $A$ have a weak conjugate $\bar{A}$. Suppose that every state of $A$ is preparable by a symmetric filter. Then $\langle a, b\rangle:=\eta_{A}\left(a, \gamma_{A}(b)\right)$ is a self-dualizing inner product on $\mathbf{E}(A)$.

Proof: Let $\alpha=\Phi(\rho)$, where $\Phi$ is a symmetric filter for some test $E$. Consider the bipartite state

$$
\omega:=\eta_{A} \circ\left(\Phi^{*} \otimes \mathrm{id}_{\bar{A}}\right)=\eta_{A} \circ\left(\operatorname{id}_{A} \otimes \bar{\Phi}^{*}\right) .
$$


For each outcome $x \in X(A)$, let $\delta_{x}$ denote the conditional state $\left(\eta_{A}\right)_{1 \mid \bar{x}}$. Then for all $x \in E$, and all outcomes $y \in X$, we have

$$
\begin{aligned}
\omega_{1 \mid \bar{x}}(y)=\frac{\eta_{A}\left(\Phi^{*}(y), \bar{x}\right)}{\eta_{A}\left(\Phi^{*}\left(u_{A}\right), \bar{x}\right)} & =\frac{\eta_{A}\left(y, \overline{\Phi^{*}}(\bar{x})\right)}{\eta_{A}\left(u_{A}, \overline{\Phi^{*}}(\bar{x})\right)} \\
& =\frac{\eta_{A}\left(y, t_{x} \bar{x}\right)}{\eta_{A}\left(u_{A}, t_{x} \bar{x}\right)} \\
& =\frac{\eta_{A}(y, \bar{x})}{\eta_{A}\left(u_{A}, \bar{x}\right)}=\left(\eta_{A}\right)_{1 \mid \bar{x}}(y)=\delta_{x}(y)
\end{aligned}
$$

It follows that $\omega_{1 \mid \bar{x}}=\delta_{x}$. It is easy to check that $\omega_{1}=\Phi\left(\left(\eta_{A}\right)_{1}\right)=\Phi(\rho)=\alpha$; also, by the law of total probability $(3), \omega_{1}=\sum_{x \in E} \omega_{2}(\bar{x}) \omega_{1 \mid \bar{x}}=\sum_{x \in E} t_{x} \delta_{x}$, where $t_{x}=\omega_{2}(\bar{x})$. Thus, every state in $\Omega(A)$ is a convex combination of the states $\delta_{x}$, and the cone generated by these states coincides with $\mathbf{V}(A)_{+}$. It follows that $\widehat{\eta}$ maps $\mathbf{E}(A)_{+}$onto $\mathbf{V}(A)_{+}$, as in the proof of Lemma 2 . The proof that $\langle a, b\rangle:=\eta(a, \bar{b})$ defines an inner product on $\mathbf{E}(A)$ now proceeds as in the proof of Lemmas 3 and 4 .

In fact, we can do a bit better:

Corollary 2 (bis) Let A have a weak conjugate, and suppose that every interior state is preparable by a reversible symmetric filter. Then $A$ is homogeneous and self-dual.

Proof: The preparability assumption clearly makes $\mathbf{V}(A)$ homogeneous. The proof of Lemma 5 shows that all states in the interior of $\Omega$ can be decomposed as in equation (4) with respect to the states $\delta_{x}=\eta_{1 \mid \bar{x}}$. As noted in Remark (2) following the proof of Theorem 1, this is enough to secure the self-duality of $\mathbf{E}(A)$, and its isomorphism with $\mathbf{V}(A)$.

It follows from the $\mathrm{KV}$ theorem that, for any model $A$ satisfying the hypotheses of either Corollary 1 or Corollary 2, $\mathbf{E}(A)$ carries a Jordan product compatible with the inner product arising from $\eta_{A}$, i.e, $\mathbf{E}(A)$ is a euclidean Jordan algebra. In fact, one can prove more: the unit effect $u$ coincides with the Jordan unit, and $\mathcal{M}(A)$ is precisely the set of Jordan frames. In other words, $\mathbf{E}(A)$ is a Jordan moel. The proof is given in Appendix $\mathrm{A}$, where it is also shown that any Jordan model satisfies the hypotheses of both corollaries. Thus, these two sets of hypotheses are equivalent, and exactly characterize the class of Jordan models. To summarize:

Theorem 2 For a finite-dimensional, uniform probabilistic model $A$, the following statements are equivalent:

(a) A is sharp, has a conjugate, and has arbitrary reversible filters

(b) A has a weak conjugate, and all non-singular states can be prepared by reversible symmetric filters

(c) A is a Jordan model.

It should be stressed that all of the assumptions going into (a) and (b) are what [7] calls singlesystem postulates, at least to the extent that the existence of a conjugate (or weak conjugate) is a property of a single system. In any event, these assumptions, whether seen as pertaining to a single system $A$ or to the pair $(A, \bar{A})$, are quite different in flavor from local tomography or the subspace axiom, which place constraints on an entire theory's worth of probabilistic models. 


\section{Conclusion}

We've seen that either of two related packages of assumptions - given in (a) and (b) of Theorem 2 - lead in a very simple way the homogeneity and self-duality of the space $\mathbf{E}(A)$ associated with a probabilistic model $A$, and hence, by the Koecher-Vinberg Theorem, to $A$ 's having a euclidean Jordan structure. While this is not the only route one can take to deriving this structure (see, e.g, [27] and [31] for approaches stressing symmetry principles), it does seem especially straightforward.

As discussed in the introduction, several other recent papers (e.g, $[20,28,14,25,11])$ have derived standard finite-dimensional quantum mechanics, over $\mathbb{C}$, from operational or informationtheoretic axioms. Besides the fact that the mathematical development here is quicker and easier, the axiomatic basis is considerably different, and arguably leaner, making no appeal to the structure of subsystems, or to the isomorphism of systems with the same information-carrying capacity, or to local tomography. The last two points are particularly important: by avoiding local tomography, we allow for real and quaternionic quantum systems; by not insisting that physical systems having the same information capacity be isomorphic, we allow for quantum theory with superselection rules, and for physical theories in which real and quaternionic systems can coexist. Of course, the door has been opened a bit wider than this: our postulates are also compatible with spin factors and with the exceptional Jordan algebra. ${ }^{10}$

Of the reconstructions cited above, the one having the strongest affinity with the approach of this paper is that of [11], the key postulate of which is that every state dilates to - that is, arise as the marginal of - a pure state on a larger, composite system, unique up to symmetries of the ancillary system. Condition (a) in the definition of a conjugate, requiring that every state dilate to a correlating state, has a somewhat similar character, albeit with the emphasis on the dilated state's correlational properties, rather than its purity. To make the connection more explicit, suppose we require that every non-singular state $\alpha$ on $A$ dilate to a correlating isomorphism state $\omega$ (which is the case, in the presence of our other assumptions). If $\mu$ is another isomorphism state with the same marginal state $\alpha$, then $\phi:=\widehat{\mu} \circ \widehat{\omega}^{-1}$ is a reversible transformation on $\mathbf{V}(\bar{A})$ with $\widehat{\mu}=\phi \circ \widehat{\omega}$, i.e., $\mu(a, b)=\omega(a, \phi(b))$ for all $a, b \in \mathbf{E}(A)$. Now, as shown in [5], if $\mathbf{V}(A)$ is irreducible as an ordered vector space, isomorphism states are pure. Thus, in the irreducible case, we have a version of the purification postulate for non-singular states. In view of these connections, it seems plausible that the approach taken here might be adapted to considerably simplify the mathematical development in [11]. ${ }^{11}$

An assumption that is common to nearly all of the cited earlier reconstructions is some version of Hardy's subspace postulate, which requires (roughly speaking) that the result of constraining a physical system to the set of states making a particular measurement-outcome impossible, also count as a physical system. This very powerful assumption, while not needed in the development above, can readily be adapted to the framework of this paper, and can to a large extent replace our assumptions above about the existence of reversible filters. The details can be found in Appendix B.

\footnotetext{
${ }^{10}$ It can be shown [6] that the exceptional Jordan algebra can be ruled out on the grounds that one can form no satisfactory composite of two euclidean Jordan algebras if either has an exceptional direct summand. Whether the spin factors can also be discarded, or whether they have some physical role to play, remains an open question.

${ }^{11}$ Going in the other direction, in [12], the authors derive a version of part (a) of our definition of a conjugate, that is, the existence of a dilation perfectly correlating two tests, from axioms similar to those of [11]. More recently, in the conext of a compact closed category of processes, the authors of [29] introduce a stronger, "symmetric" version of the purification postulate, and show that when combined with suitable versions of sharpness and the existence of a "classical interface", this implies that all states can be prepared from the maximally mixed state by a reversible process, allowing them to prove that their analogue of the cone $\mathbf{V}(A)_{+}$is homogeneous and self-dual.
} 
It is worth remarking that the subspace axiom applies, not to an indidual probabilistic model but to a class of probabilistic models, that is, to an entire probabilistic theory. (In the language of [7], it is not a single-system postulate.) As a rule, one wants to think of a physical theory, not as a loosely structured class, but as a category of systems, with morphisms corresponding to processes. To allow for composite systems, it is natural to take this to be a symmetric monoidal category [1]. This brings us to the interesting question of whether one can construct symmetric monoidal categories of probabilistic models, in which (say) the hypotheses of Corollary 1 are satisfied by all systems. This is indeed possible for special Jordan algebras (those not having the exceptional Jordan algebra as a direct summand). Restricting attention to Jordan models corresponding to direct sums of real, complex and quaternionic matrix algebras, one can even arrange for this category to be compact closed [5]. This implies that many standard quantum-information theoretic protocols, notably conclusive teleportation and entanglement-swapping, are still available in this non-locally tomographic setting. ${ }^{12}$

Acknowledgements I wish to thank Giulio Chiribella, Chris Heunen, Matt Leifer and Markus Müller for helpful comments on earlier drafts of this paper. This work was supported in part by a grant (FQXi-RFP3-1348) from the FQXi foundation.

\section{References}

[1] S. Abramsky and B. Coecke, Categorical quantum mechanics, in D. Gabbay, K. Engesser and D. Lehman, Handbook of Quantum Logic and Quantum Structures vol II, Elsevier, 2008; DOI:10.1016/B978-0-444-52869-9.5001-4; arXiv: quant-ph/0402130)

[2] E. Alfsen and F. Shultz, Geometry of state spaces of operator algebras, Birkhäuser, 2003 DOI: 10.1007/978-1-4612-0019-2

[3] C. Aliprantis and D. Toukey, Cones and Duality, Springer, 2007 DOI: 10.1090/gsm/084

[4] J. Baez, Division algebras and quantum theory, Foundations of Physics 42 819-855 (2012) DOI: 10.1007/s10701-011-9566-z; arXiv:1101.5690

[5] H. Barnum, C. Gaebler and A. Wilce, Ensemble steering, weak self-duality and the structure of probabilistic theories, Foundations of Physics 43 1411-1437 (2013) doi:10.1007/s10701-0139752-2; arXiv:0912.5532

[6] H. Barnum, M. Graydon and A. Wilce, Some Nearly Quantum Theories, in C. Heunen, P. Selinger and J. Vicary, eds., Proceedings of the 12th International Workshop on Quantum Physics and Logic, EPTCS 195 (2015), 59-70 10.4204/EPTCS.195.5; arXiv:1507.06278

[7] H. Barnum, M. Mueller and C. Ududec, Higher-order interference and single-system postulates characterizing quantum theory, New Journal of Physics 16 (2014) DOI: 10.1088/13672630/16/12/123029; arXiv:1403.4147

\footnotetext{
${ }^{12}$ More generally, the existence of conjugate systems is very suggestive of the "caps" that define part of a compact structure on a symmetric monoidal category (an observation reflected in my choice of the notation $\eta$ ). For a further development of this connection, see [32]. See also [29] for a reconstruction of finite-dimensional quantum theory from a "symmetric purification" postulate on the structure of a suitable dagger-monoidal category allowing "classical control".)
} 
[8] H. Barnum and A. Wilce, Local tomography and the Jordan structure of quantum theory, Found. Phys. 44 (2014), 192-212 DOI: 10.1007/s10701-014-9777-1; arXiv:1202.4513

[9] H. Barnum and A. Wilce, Post-classical probability theory, in G. Chiribella and R. Spekkens, eds., Quantum Theory: Informational Foundations and Foils, Springer, 2017 doi:10.1007/97894-017-7303-4_11; arXiv:1205.3833

[10] J. Barrett, Information processing in generalized probabilistic theories, Physical Review A 75 (2005) DOI: 10.1103/PhysRevA.75.032304; arXiv:quant-ph/0508211

[11] G. Chiribella, M. D'Ariano and P. Perinotti, Informational derivation of quantum theory, Physical Review A 84 (2011), 012311 DOI: 10.1103/PhysRevA.84.012311; arXiv: 1011.6451

[12] G. Chiribella and C. M. Scandolo, Operational axioms for diagonalizing states, in C. Heunen, P. Selinger and J. Vicary, Proceedings of the 12th International Workshop on Quantum Physics and Logic, EPTCS 195 (2015) 96-115 DOI: 10.4204/EPTCS.195.8; arXiv:1608. 04459

[13] B. Coecke and A. Kissinger, Categorical Quantum Mechanics I: causal quantum processes, in E. Landry, ed., Categories for the Working Philosopher, Oxford, 2017 DOI: 10.1093/oso/9780198748991.003.0012; arXiv: 1510.05468

[14] B. Dakic and C. Brukner, Quantum theory and beyond: is entanglement special? in H. Halvorson, ed., Deep Beauty, Cambridge, 2011 DOI: 10.1017/CBO9780511976971; arXiv:0911.0695

[15] E. B. Davies and J. Lewis, An operational approach to quantum probability, Communications in Mathematical Physics 17 (1970) 239-260 DOI: 10.1007/BF01647093

[16] C. M. Edwards, The operational approach to algebraic quantum theory I, Communications in Mathematical Physics 16 (1970), 207-230 DOI: 10.1007/bf01646788

[17] D. J. Foulis and C. H. Randall, Empirical logic and tensor products, in H. Neunmann (ed.), Foundations of Interpretations and Foundations of Quantum Mechanics, B.I.Wissenshaftsverlag, 1981

[18] J. Faraut and A. Korányi, Analysis on Symmetric Cones, Oxford, 1994

[19] H. Hanche-Olsen, JB algebras with tensor products are $C^{*}$ algebras, in H. Araki et al. (eds.), Operator Algebras and their Connections with Topology and Ergodic Theory, Lecture Notes in Mathematics 1132 (1985), 223-229 DOI: 10.1007/BFb0074886

[20] L. Hardy, Quantum theory from five reasonable axioms, arXiv:quant-ph/0101012, 2001

[21] P. Janotta and R. Lal, Generalized probabilistic theories without the no-restriction hypothesis, Physical Review A. 87 (2013) DOI: 10.1103/PhysRevA.87.052131; arXiv:1302.2632

[22] P. Jordan, J. von Neumann and E. Wigner, On an algebraic generalization of the quantum mechanical formalism, Annals of Mathematics 35 (1934), 29-64 DOI: 10.2307/1968117 
[23] M. Koecher, The Minnesota Notes on Jordan Algebras and their Applications, Ed. A. Krieg and S. Walcher, Springer Lecture Notes in Mathematics 1710, Springer, 1999 DOI: 10.1007/BFb0096285

[24] G. Ludwig, Foundations of Quantum Mechanics, Springer, 1983 DOI: 10.1007/978-3-64286751-4

[25] Ll. Masanes and M. Müller, A derivation of quantum theory from physical requirements, New Journal of Physics 13 (2011) DOI: 10.1088/1367-2630/13/6/063001; arXiv:1004.1483

[26] M. Mueller and Ll. Masanes, Information-theoretic postulates for quantum mechanics, in G. Chiribella and R. Spekkens, eds. Quantum Theory: Informational Foundations and Foils, Springer, 2016 doi:10.1007/978-94-017-7303-4_5; arXiv: 1203.451

[27] M. Müller and C. Ududec, The structure of reversible computation determines the selfduality of quantum theory, Physical Review Letters 108 (2012), 130401 DOI: 10.1103/PhysRevLett.108.130401; arXiv:1110.3516

[28] J. Rau, On quantum vs. classical probability, Annals of Physics 324 (2009) 2622-2637 DOI: 10.1016/j.aop.2009.09.013; arXiv:0710.2119

[29] J. Selby, C. M. Scandolo and B. Coecke, Reconstructing quantum theory from diagrammatic postulates, arXiv: 1802.00367

[30] A. Wilce, Four and a half axioms for finite-dimensional quantum theory in Y. Ben-Menahem and M. Hemmo (eds.), Probability in Physics, Springer, 2012 doi:10.1007/978-3-642-213298_17; arXiv:0912.5530

[31] A. Wilce, Symmetry, self-duality and the Jordan structure of finite-dimensional quantum theory, DOI: 10.4204/EPTCS.95.19; arXiv:1210.0622

[32] A. Wilce, A shortcut from categorical quantum mechanics to convex operational theories, in B. Coecke and A. Kissinger (Eds.), 14th International Conference on Quantum Physics and Logic (QPL), EPTCS 266 (2018) 222-236; DOI: 10.4204/eptcs.266.15; arXiv:1206.2897

\section{A Jordan Models}

Let $\boldsymbol{J}$ be a euclidean Jordan algebra. As discussed earlier, this is associated with a probabilistic model $A(\boldsymbol{J})=(X(\boldsymbol{J}), \mathcal{M}(\boldsymbol{J}), \Omega(\boldsymbol{J}))$, where $X(\boldsymbol{J})$ is the set of primitive idempotents (that is, minimal projections) in $\boldsymbol{J}, \mathcal{M}(\boldsymbol{J})$ is the set of Jordan frames (maximal pairwise orthogonal sets of minimal projections), and $\Omega(\boldsymbol{J})$ is the set of states on $(X(\boldsymbol{J}), \mathcal{M}(\boldsymbol{J}))$ arising from states on $\boldsymbol{J}$, that is, restrictions to $X(\boldsymbol{J})$ of positive, normalized linear functionals on $\boldsymbol{J}$. Using the self-duality of $\boldsymbol{J}$, it's easy to show that $\mathbf{E}(A) \simeq \boldsymbol{J} \simeq \mathbf{V}(A)$.

In this appendix, it is shown that any probabilistic model satisfying the conditions of Corollary 1 is actually a Jordan model of this form, and, conversely, that any such Jordan model satisfies the hypotheses of Corollary 2 (which imply those of Corollary 1 ). 


\section{A.1 Direct Sums and central projections}

At several points, we will need to use some basic facts about direct sum decompositions of Jordan algebras. The direct sum of Jordan algebras $\boldsymbol{J}_{1}, \ldots, \boldsymbol{J}_{n}$ is the algebraic direct sum $\boldsymbol{J}_{=}=\boldsymbol{J}_{1} \oplus \cdots \oplus \boldsymbol{J}_{n}$ of the vector spaces $\boldsymbol{J}_{i}$, consisting of $n$-tuples $\left(a_{1}, \ldots, a_{n}\right)$ with $a_{i} \in \boldsymbol{J}_{i}$, with the Jordan product defined by $\left(a_{i}\right) \bullet\left(b_{i}\right)=\left(a_{i} \bullet{ }_{i} b_{i}\right)$, where $\bullet_{i}$ is the Jordan product on the $i$-th summand. Identifying $a \in \boldsymbol{J}_{i}$ with $\left(a_{i}\right)$ where $a_{j}=0$ for $j \neq i$ and $a_{j}=a$ for $j=i$, we can treat each $\boldsymbol{J}_{i}$ as a subalgebra of $\boldsymbol{J}$, and write $\left(a_{i}\right) \in \boldsymbol{J}$ as $\sum_{i=1}^{n} a_{i}$. With this understood, we have $a \bullet b=0$ if $a \in \mathbf{E}_{i}$ and $b \in \mathbf{E}_{j}$ with $i \neq j$. The unit is evidently $\mathbf{1}=\sum_{i=1}^{n} \mathbf{1}_{i}$ where $\mathbf{1}_{i}$ is the unit in $\boldsymbol{J}_{i}$. Note that the canonical projection map $\pi_{i}: \boldsymbol{J} \rightarrow \boldsymbol{J}_{i}$ is a Jordan homomorphism. Thus, $e \in \mathbf{E}$ is an idempotent iff $e_{i}:=\pi(e)$ is an idempotent in $\boldsymbol{J}_{i}$. As $e=\sum_{i} e_{i}$, it follows that $e$ is a primitive idempotent iff $e=e_{i} \in \mathbf{E}_{i}$ for some $i=1, \ldots, n$. In other words, every primitive idempotent of $\boldsymbol{J}$ lives in one of the summands $\boldsymbol{J}_{i}$.

If each $\boldsymbol{J}_{i}$ is a euclidean Jordan algebra with inner product $\langle,\rangle_{i}$, then we endow $\boldsymbol{J}=\bigoplus_{i} \boldsymbol{J}_{i}$ with the usual inner product, that is, $\langle a, b\rangle=\sum_{i}\left\langle a_{i}, b_{i}\right\rangle_{i}$. where $a_{i}=\pi_{i}(a)$ and $b_{i}=\pi_{i}(b)$. Thus, the summands $\boldsymbol{J}_{i}$ are orthogonal to one another as subspaces of $\boldsymbol{J}$. A euclidean Jordan algebra is simple iff it is not isomorphic to a direct sum of non-trivial Jordan algebras. Every EJA is isomorphic to a direct sum of simple EJAs in an essentially unique way. It will be helpful briefly to review how this decomposition works. Elements $a$ and $b$ in an EJA $\boldsymbol{J}$ operator commute with one another iff $a \bullet(b \bullet x)=b \bullet(a \bullet x)$ for every $x \in \boldsymbol{J}$, i.e., iff the operators $L_{a}$ and $L_{b}$ of left Jordan-multiplication by $a$ and $b$ commute. An element of $\boldsymbol{J}$ is central iff it operator-commutes with all elements of $\boldsymbol{J}$. If $\boldsymbol{J}=\bigoplus_{i} \boldsymbol{J}_{i}$, then each of the units $\mathbf{1}_{i} \in \boldsymbol{J}_{i}$ is central. Note that these elements are also idempotents, or projections. A Jordan ideal of an EJA $\boldsymbol{J}$ is a subspace of the form $c \boldsymbol{J}$ where $c$ is a central projection, which is then unique. In this case, the mapping $x \mapsto c \bullet x$ is a Jordan homomorphism from $\boldsymbol{J}$ onto $c \boldsymbol{J}$; indeed, $x \mapsto\left(c \bullet x, c^{\prime} \bullet x\right)$ provides a canonical isomorphism $\boldsymbol{J} \simeq c \boldsymbol{J} \oplus c^{\prime} \boldsymbol{J}$. More generally, recall that idempotents $e, f$ in a Jordan algebra are Jordan-orthogonal iff $e \cdot f=0$. If $\left\{c_{i}\right\}$ is a maximal pairwise Jordan-orthogonal set of central idempotents, then it is straightforward to show that $\sum_{i} c_{i}=\mathbf{1}$ and, hence, that $\boldsymbol{J} \simeq \bigoplus c_{i} \boldsymbol{J}$ via the mapping $\phi: a \mapsto\left(c_{i} a\right)$. Moreover, each of the summands $\boldsymbol{J}_{i}:=c_{i} \boldsymbol{J}$ is simple. For if $c_{i}=p+q$ where $p$ and $q$ are central projections in $c_{i} \boldsymbol{J}$, then $p, q$ are central in $\boldsymbol{J}$, and are Jordan-orthogonal to every $c_{j}$ with $j \neq i$, so $\left\{c_{i}\right\}_{i \neq j} \cup\{p, q\}$ is a larger pairwise Jordan-orthogonal set of central projections, a contradiction.

\section{A.2 The unit effect as the Jordan unit}

Suppose $A$ is a model satisfying the hypotheses of Corollary 1. In particular, then, $A$ is HSD, so $\mathbf{E}:=\mathbf{E}(A)$ has a Jordan structure. We wish to show that the unit effect $u \in \mathbf{E}(A)$ is (or can be taken to be) the Jordan unit, and that each outcome $x \in X(A)$ - or, more exactly, the corresponding effect $\widehat{x}$ - is a primitive idempotent. ${ }^{13}$ In fact, we will ultimately establish more, namely, that $X(A)$ corresponds exactly to the set of primitive idempotents, and $\mathcal{M}(A)$, to the set of Jordan frames, of $\mathbf{E}(A)$.

In what follows, normalize the inner product on $\mathbf{E}$ so that $\langle x, x\rangle=1$ for every $x \in X(A)$. (This is possible, since the inner product arising from a correlator assigns every outcome the same norm.) Note that we also have $\langle u, x\rangle=1$, and $\langle u, u\rangle=n$, the rank of $A$. Every state $\alpha \in \mathbf{V}(A)$ corresponds to a unique $a \in \mathbf{E}(A)_{+}$with $\alpha(x)=\langle a, x\rangle$. In particular, $\langle a, u\rangle=1$. Conversely,

\footnotetext{
${ }^{13}$ This can actually be established very directly by appealing to a slightly stronger version of the Koecher-Vinberg Theorem, as in [8]. The proof given here is more self-contained and elementary.
} 
every $a \in \mathbf{E}(A)_{+}$with $\langle a, u\rangle=1$ corresponds to a state in this way, since $\mathbf{E}(A) \simeq \mathbf{V}(A)$ as ordered spaces.

The KV theorem produces a Jordan structure on $\mathbf{E}(A)$ in which the Jordan unit, $\mathbf{1}$, is fixed by every order-automorphism that is also an orthogonal transformation relative to the inner product. That is, writing $\operatorname{Aut}(\mathbf{E})$ for the group of order-automorphisms and $\operatorname{Aut}(\mathbf{E})_{1}$ for the stabilizer of $\mathbf{1}$ therein, $\operatorname{Aut}(\mathbf{E}) \cap O(\mathbf{E}) \subseteq \operatorname{Aut}(\mathbf{E})_{\mathbf{1}}$. Moreover, the stabilizer of $\mathbf{1}$ in the connected component $G$ of Aut $(\mathbf{E})$ is then exactly $K:=G \cap S O(\mathbf{E})$ [18]. In particular, every order-automorphism in the connected component of the identity fixing $\mathbf{1}$ is an orthogonal transformation with respect to $\langle$,$\rangle .$

In the interest of notational simplicity, from this point on I will identify outcomes $x \in X(A)$ with the corresponding evaluation functionals $\widehat{x} \in \mathbf{E}(A)$, treating each test $E \in \mathcal{M}(A)$ as a set of effects.

A.1 Lemma: For each $x \in X(A)$, there exists a primitive idempotent $e_{x}$ and a scalar $t_{x}>0$ such that $x=t_{x} e_{x}$, and every primitive idempotent corresponds to an outcome in this way.

Proof: Since $A$ is sharp and $\langle x, x\rangle=\langle x, u\rangle=1$, we have $\delta_{x}=\langle x|$ for every $x \in X(A)$; that is, $\delta_{x}(a)=\langle x, a\rangle$ for all $a \in \mathbf{E}(A)$. In particular, as $\delta_{x}$ is a pure state, $x$ is ray-extremal for every $x \in X(A)$. Hence, $x=t_{x} e_{x}$ for some primitive idempotent $e_{x}$. Conversely, since $X(A)$ generates $\mathbf{E}(A)$, every ray-extremal element of $\mathbf{E}(A)_{+}$must be a multiple of some $x \in X(A)$. In particular, then, each primitive idempotent is a multiple of an outcome, and vice versa.

Notice that if $x$ and $y$ are distinct outcomes belonging to a common test, we have $\left\langle e_{x}, e_{y}\right\rangle=$ $\frac{1}{t_{x} t_{y}}\langle x, y\rangle=0$. Hence, $1=\langle x, x\rangle=t_{x}^{2}\left\|e_{x}\right\|^{2}$, so $t_{x}=1 /\left\|e_{x}\right\|$ for every $x$.

A.2 Theorem: The Jordan product on $\mathbf{E}(A)$ can be so chosen that $u_{A}$ is the Jordan unit, $\mathbf{1}$ and every $x \in X(A)$ is a primitive idempotent.

Proof: We consider in turn the case in which $\mathbf{E}(A)$ is simple and the general case in which $\mathbf{E}(A)$ is a direct sum of simple ideals. Throughout, we write $u$ for $u_{A}$.

Case 1: $\mathbf{E}(A)$ is simple. By [18] Corollary IV.2.7, the group $K:=G \cap S O(\mathbf{E})$, where $G$ is the connected component of the identity in $\operatorname{Aut}(\mathbf{E})$, acts transitively on the set of primitive idempontents. Since $K$ consists of orthogonal transformations, all primitive idempotents have the same norm. It follows that, for $\mathbf{E}$ simple, $\|e\| \equiv c>0$ for all primitive idempotents, whence, that we have $t_{x} \equiv t=1 / c$ for all $x$. That is, if $\mathbf{E}(A)$ is simple, $x=t e_{x}$ for every $x \in X(A)$. Now redefine the Jordan product on $\mathbf{E}$ by setting $a \circ b:=t^{-1} a \bullet b$. It is easy to check that this gives a Jordan product defining the same positive cone, with unit $\mathbf{1}^{\prime}:=t \mathbf{1}$. Also note that the new Jordan product continues to ineract with the given innner product in the desired way, i.e., $\langle a \circ b, c\rangle=\langle b, a \circ c\rangle$ for all $a, b, c \in \mathbf{E}$. We also have, for each outcome $x \in X(A)$,

$$
x \circ x=t^{-1}\left(t e_{x} \bullet t e_{x}\right)=t\left(e_{x} \bullet e_{x}\right)=t e_{x}=x,
$$

so $x$ is an idempotent with respect to this new Jordan product; moreover, since the positive cone is unchanged, this is still ray-extremal, hence, a primitive idempotent. We now have

$$
\left\langle x, \mathbf{1}^{\prime}\right\rangle=\left\langle x^{2}, \mathbf{1}^{\prime}\right\rangle=\langle x, x\rangle=1 .
$$

By the spectral decomposition for states (equation (4)), we have $\mathbf{1}^{\prime}=\sum_{x \in E} s_{x} x$ for some test 
$E \in \mathcal{M}(A)$ and coefficients $s_{x}$. But for every $x \in E$,

$$
s_{x}=\sum_{y \in F} s_{y}\langle x, y\rangle=\left\langle x, \mathbf{1}^{\prime}\right\rangle=1
$$

so $\mathbf{1}^{\prime}=\sum_{x \in E} x=u_{A}$.

Case 2: $\mathbf{E}(A)$ a direct sum of simple ideals. Let $\mathbf{E}=\bigoplus_{i=1}^{k} \mathbf{E}_{i}$ where each $\mathbf{E}_{i}$ is a simple Jordan algebra. By Lemma A.1, we still have a correspondence $x \mapsto e_{x}$ between outcomes $x \in X(A)$ and primitive idempotents $e_{x} \in \mathbf{E}$, with $x=t_{x} e_{x}$ for some $t_{x}>0$. As remarked earlier, each primitive idempotent lies in a unique summand $\mathbf{E}_{i}$ whence, the same is true for outcomes. The argument given above shows that, for all $x \in \mathbf{E}_{i}$, we have $t_{x}=t_{i}$ for a constant $t_{i}>0$ depending only on the summand. Adjusting the Jordan product on each summand as in Case 1, we obtain a new Jordan product on $\mathbf{E}$ given by

$$
\left(a_{i}\right) \circ\left(b_{i}\right)=\sum_{i} t_{i}^{-1} a_{i} \bullet b_{i}
$$

rendering each $x \in X(A) \cap \mathbf{E}_{i}$ - and hence, each outcome $x \in X(A)$ - a primitive idempotent. By the same argument as in Case 1, we have $\langle x, \mathbf{1}\rangle=1$ for all $x \in X$. Expanding $\mathbf{1}=\sum_{x \in E} s_{x} x$ for some test $E \in \mathcal{M}(A)$, we then have (again, just as in the irreducible case) that $\left.\left.1=\langle x, \mathbf{1}\rangle=\sum_{y \in E} s_{y}\right\rangle x, y\right\rangle=s_{x}$, whence, $s_{x}=1$ for all $x \in E$, and hence, $\mathbf{1}=u_{A}$.

From now on, we treat $u_{A}$ as the Jordan unit of $\mathbf{E}(A)$ without further comment, and revert to writing $u$, rather than $\mathbf{1}$, for the unit in an abstract Jordan algebra. It will be convenient at this point to adopt the notation $x \perp y$ to indicate that two outcomes $x, y \in X(A)$ are distinct and belong to a common test.

A.3 Corollary: Let $x, y \in X(A)$. Then $x \perp y \Rightarrow x \bullet y=0$.

Proof: By [2], Prop. 2.18, if $p, q$ are projections in an EJA, then $p \leq u-q$ implies $p \bullet q=0$. By Proposition A.2, $x$ and $y$ are projections. If $x \perp y$, then $x+y \leq u$, so $x \leq u-y$.

Idempotents $p, q$ in a Jordan algebra are Jordan-orthogonal iff $p \bullet q=0$. Recall that a Jordan frame in an EJA $\mathbf{E}$ is a set $E$ of non-zero Jordan-orthogonal primitive idempotents summing to the unit. It follows from Proposition A.2 and Corollary A.3 that every test in $\mathcal{M}(A)$ defines a Jordan frame in $\mathbf{E}(A)$. We now show that, conversely, every Jordan frame in $\mathbf{E}(A)$ belongs to $\mathcal{M}(A)$.

A.4 Theorem: Let $A$ be any probabilistic model satifsying the hypotheses of Corollary 1 or of Corollary 2, and let $\mathbf{E}(A)$ have the Jordan structure in which $u_{A}$ is the Jordan unit, as per Lemma A.2. Then every Jordan frame of $\mathbf{E}(A)$ corresponds to a test in $\mathcal{M}(A)$. Hence, $A$ is a Jordan model.

Proof: Theorem A.2 and Corollary A.3 tell us that the set $X(A)$ of outcomes of $A$ is exactly the set of primitive idempotents (still continuing to identify outcomes with the corresponding effects), and that every test $E \in \mathcal{M}(A)$ is a Jordan frame of $\mathbf{E}(A)$. The Spectral Theorem for euclidean Jordan algebras ([18] Theorem III.1.1) tells us that if $\boldsymbol{J}$ is any EJA with unit $u$ and $a \in \boldsymbol{J}$, then there exists a unique family of mutually Jordan-orthogonal non-zero idempotents $e_{i}$ and distinct coefficients $s_{i}$, such that $a=\sum_{i} s_{i} e_{i}$. Suppose the idempotents $e_{i}$ are primitive, and that $a$ can 
also be expanded as $\sum_{j} t_{j} f_{j}$ for some pairwise Jordan-orthogonal non-zero idempotents $f_{j}$ and coefficients $t_{j}$ (not a priori distinct). Then by collecting those terms with common coefficients, we can also write $a=\sum_{k} k p_{k}$ where $p_{k}=\sum\left\{f_{j} \mid t_{j}=k\right\}$. Since the $f_{j}$ are pairwise Jordan-orthogonal idempotents, it is easy to check that $p_{k}$ is also an idempotent, with $p_{k} \bullet p_{k^{\prime}}=0$ for $k \neq k^{\prime}$. It follows from the uniqueness of the spectral expansion that, for each $k$, there is some $i$ with $k=s_{i}$ and $p_{k}=e_{i}$. Since $e_{i}$ is primitive, this means that the sum $p_{k}=\sum\left\{f_{j}: t_{j}=k\right\}$ has a unique term, call it $f_{j_{i}}$, with $f_{j_{i}}=e_{i}$. It follows that the coefficients $t_{j}$ were after all distinct, and the projections $f_{j}$ coincide with the projections $e_{i}$. In other words, if $a$ can be expanded with dinstinct coefficients relative to some family of mutually orthogonal primitive idempotents, it can have no other such expansion in terms of mutually orthogonal idempotents, with any coefficients. Now let $F=\left\{e_{1}, \ldots, e_{n}\right\}$ be a Jordan frame of $\mathbf{E}(A)$. Choosing distinct coefficients $s_{i}, i=1, \ldots, n$, let $a=\sum_{i} t_{i} e_{i}$. By our spectral decomposition for HSD models (equation (4)), there exists a test $E \in \mathcal{M}(A)$ and coefficients $t_{x}, x \in E$ such that $a=\sum_{x \in E} t_{x} x$. Since $E$ is a Jordan frame, the uniqueness result above tells us that $E=F$. Thus, every Jordan frame of $\mathbf{E}(A)$ is a test in $\mathcal{M}(A)$, as advertised.

\section{A.3 Conjugates and Filters for Jordan Models}

As discussed in Section 5, the question of what it means, physically, to say that a given system has a conjugate really depends on the probabilistic theory - and the notion of composite system - with which one is concerned. But if we are content to interpret this idea very broadly, we can understand the composite $A \bar{A}$ as the "maximal" tensor product $A \otimes \max \bar{A}$ [9], the states of which are simply the positive, normalized bilinear forms on $\mathbf{E}(A) \times \mathbf{E}(\bar{A})$. In particular, if $A=A(\boldsymbol{J})$ is the model associated with a euclidean Jordan algebra $\boldsymbol{J}$, the canonical inner product on $\boldsymbol{J}$, normalized so that $\langle u, u\rangle=1$, supplies exactly the needed bilinear form. Thus, every Jordan model can be regarded as its own weak conjugate. (At least, this is so if we construe " $A$ has a weak conjugate" to mean only that there exists a perfectly correlating positive, bilinear form on $\mathbf{E}(A)$ - mathematically, a weaker condition than that $A$ have a conjugate in any particular probabilistic theory.)

We now show that Jordan models support arbitrary filters, and that every state of such a model can be prepared by a symmetric filter. For any element $a$ of a euclidean Jordan algebra $\boldsymbol{J}$, the operator $U_{a}: \boldsymbol{J} \rightarrow \boldsymbol{J}$ defined by

$$
U_{a}(x):=2 a \cdot(a \cdot x)-a^{2} \cdot x
$$

is positive ([2] Theorem 1.25). If $\boldsymbol{J}$ is special, i.e., if $\boldsymbol{J}$ consists of self-adjoint operators on a real, complex or quaternionic Hilbert space $\mathcal{H}$, with $a \cdot b=\frac{1}{2}(a b+b a)$, one can check that $U_{a}(x)=a x a$. Below, we shall see that if $a=\sum_{x \in E} t_{x} x$, where $E$ is a Jordan frame, then $U_{a}$ is a filter with coefficients $t_{x}$.

Let $\boldsymbol{J}$ be an EJA. An element $a \in \boldsymbol{J}$ is invertible iff there exists an element $b$ of the associative sub-algebra generated by $a$ and $u$, such that $a \bullet b=u$. This element, which is clearly unique, is then the inverse of $a$, denoted by $a^{-1}$. The following collects some facts about invertibility that will be needed in a number of places below.

A.5 Proposition: The following are equivalent:

(a) a is invertible;

(b) There exists some $b \in \boldsymbol{J}$ with $a \bullet b=u$ and $a^{2} \bullet b=a$;

(c) $U_{a}$ is invertible. In this case, $U_{a}^{-1}=U_{a^{-1}}$. 
Proof: That (a) implies (b) is clear. That (b) implies (a) is a consequence of the fact (the ShirsovCohn Theorem; see [2], Proposition 1.14) that the Jordan algebra generated by two elements and $u$ is special, plus the fact that (a) and (b) are equivalent in special Jordan algebras. See [2] Lemma 1.16 and Proposition 1.17 for details. The equivalence of (a) and (c) is Lemma 1.23 in [2].

We shall also need the following elementary observation:

A.6 Lemma: If a has a spectral decomposition $a=\sum_{x \in E} t_{x} x$ where $E$ is a Jordan frame and $t_{x} \neq 0$ for all $x \in E$, then $a$ is invertible. In particular, $a$ is invertible if lies in the interior of $\boldsymbol{J}_{+}$.

Proof: By spectral theory, $a=\sum_{x \in E} t_{x} x$ where $E$ is a Jordan frame. If $t_{x} \neq 0$ for all $x$, let $b=\sum_{x \in E} t_{x}^{-1} x=f(a)$ where $f(x)=x^{-1}$, so that $b \in C(a, u)$, the Jordan subalgebra generated by $a$ and $u$. Observe that, by the Jordan-orthogonality of elements of a Jordan frame,

$$
a \cdot b=\sum_{x, y \in E} t_{x} t_{y}^{-1} x \cdot y=\sum_{x} t_{x} t_{x}^{-1} x=\sum_{x \in E} x=u .
$$

Thus $a$ is invertible with inverse $b$.

A.7 Lemma: Let $\boldsymbol{J}$ be any EJA. Every state of the model $A(\boldsymbol{J})$ is preparable by symmetric filter, and every non-singular state, by a reversible symmetric filter.

Proof: Let $\alpha$ be any state on $\boldsymbol{J}$. By self-duality, there exists a unique $w \in \boldsymbol{J}_{+}$with $\alpha(b)=\langle w, b\rangle$ for all $b \in \boldsymbol{J}$. The spectral theorem gives us a Jordan frame $E$ and a decomposition $w=\sum_{x \in E} t_{x} x$. Let $a=\sum_{x \in E} t_{x}^{1 / 2} x \in \boldsymbol{J}_{+}$: then we have $U_{a}(u)=w$ and $U_{a}(x)=t_{x} x$ for every $x \in E$, so $U_{a}$ is a filter. Since left multiplication by $a$ is self-adjoint with respect to the inner product on $\boldsymbol{J}$, so is $U_{a}$, whence, $U_{a}$ is a symmetric filter. Finally, if $w$ lies in the interior of $\boldsymbol{J}_{+}$, then the coefficients $t_{x}$, and hence also $t_{x}^{1 / 2}$, are all strictly positive. Thus, $a$ is invertible by Lemma A.5, and thus $U_{a}$ is invertible with inverse $U_{a^{-1}}$, again a positive operator, by Proposition A.5.

As noted in the discussion preceding Lemma 5, every state preparable by a symmetric reversible filter is the marginal of a correlating state. Hence, every Jordan model is its own (strong) conjugate. Since such a model is evidently sharp, the conditions of Corollary 1 are satisfied. This proves Theorem 2: every probabilistic model satisfying the hypotheses of Corollary 1 or Corollary 2 is a Jordan model, and every Jordan model satisfies the hypotheses of both Corollaries.

\section{B Symmetry and Subspace Axioms}

In most other recent reconstructions of $\mathrm{QM}[20,14,25,11]$, one encounters some version of a subspace axiom. Informally, the idea is that if we constrain the states of a given system so as to render a certain measurement result impossible, we obtain what amounts the state space of a new system in its own right, to which any other axioms must then apply. In practice, the subsets of the state space arising in this way are (some of the) faces of the larger state space ${ }^{14}$. An assumption of this sort was made by Hardy [20], and, following his lead, is also central in reconstructions by Dakic and Brukner [14], Masanes and Mueller [25] and Chiribella, D'Ariano and Perinotti [11], although the precise formulation varies somewhat from one set of authors to another.

\footnotetext{
${ }^{14}$ Recall that a face of a convex set $K$ is a convex set $F \subseteq K$ such that for all $\alpha, \beta \in K$ and all $t \in(0,1)$, $t \alpha+(1-t) \beta \in F$ implies $\alpha, \beta \in F$.
} 
In this Appendix it is established that, for uniform probabilistic models, a certain version of this subspace axiom, plus a very reasonable compactness assumption, enforce a spectral decomposition of states (equation (4) in Section 3). Thus, these assumptions can replace condition (b) in the definition of a conjugate, at least as far as Theorem 1 is concerned. Moreover, a stronger version of the subspace axiom, which seems about equally well motivated, implies the existence of arbitrary reversible filters. Combined with a rather weak and operationally natural symmetry assumption, this in turn yields the homogeneity of the space $\mathbf{V}(A)$. In the presence of weak conjugates, it follows that $\mathbf{V}(A)$ is both homogeneous and self-dual.

Probabilistic Theories Before proceeding, it will be important to clarify the term "probabilistic theory", up to this point used rather freely and informally. In the very broadest sense, a probabilistic theory is simply a class of probabilistic models, together with some designated processes that are singled out for study. But allowing for the composition of processes, and assuming that identity operators count as processes, it is very natural to assume that a probabilistic theory is a category of probabilistic models and processes. This is the point of view taken in the "categorical quantum mechanics" programme of Abramsky, Coecke and others [1]. It is also usual in this context also to assume that this category has a (symmetric) monoidal structure, allowing for the formation of composite systems. However, for my purposes, it will be enough to require only that we are given a class $\mathcal{C}$ of probabilistic models and, for each model $A \in \mathcal{C}$, a preferred monoid $\operatorname{Proc}(A)$ of allowed processes, i.e., positive linear mappings $T: \mathbf{V}(A) \rightarrow \mathbf{V}(A)$ with $u_{A}(T(\alpha)) \leq 1$ for all $\alpha \in \Omega(A) .{ }^{15}$

We continue to identify outcomes with the corresponding effects, regarding $X(A)$ as a subset of $\mathbf{E}(A)$ and $\mathcal{M}(A)$, as a collection of subsets of $\mathbf{E}(A)$. We can then define a symmetry of $A$ to be a dual process $g=\phi^{*}$ where $\phi \in \operatorname{Proc}(A)$ is invertible, such that $g \mathcal{M}(A)=\mathcal{M}(A)$. I will write $G(A)$ for the set of all symmetries of $A$, noting that this is a group. As above, we use the notation $x \perp y$ to indicate that $x$ and $y$ are distinct outcomes belonging to a common test. Notice that this does not (yet) imply that $x$ and $y$ are orthogonal with respect to any inner product on $\mathbf{E}(A)$.

\section{B.1 The Subspace Axiom and Spectrality}

Masanes and Mueller's Framework In the reconstruction due Masanes and Mueller [25], one begins by specifying the state-space of a physical system, taken to be a finite-dimensional compact convex set $\Omega$. Measurement results are associated with effects, i.e., affine functionals $a: \Omega \rightarrow \mathbb{R}$ with $0 \leq a(\alpha) \leq 1$ for all $\alpha \in \Omega$. Of course, $a(\alpha)$ is understood as the probability of the result associated with $a$ being obtained, when the system's state is $\alpha$. Masanes and Mueller do not assume that all effects correspond to physically realizable measurement results, but do seem, tacitly, to define a measurement to be any list $a_{1}, \ldots, a_{n}$ of allowed effects that sum to the unit effect $u$ (where $u(\alpha)=1$ for all $\alpha \in \Omega(A)$ ). Further assumptions, explicit in [26], are that the set of allowed effects is topologically closed, convex, and closed under $a \mapsto u-a$. Masanes and Mueller call states $\alpha_{1}, \ldots, \alpha_{n} \in \Omega(A)$ perfectly distinguishable iff there exist affine functionals $a_{i}: \Omega \rightarrow \mathbb{R}$ with $0 \leq a_{i}(\alpha) \leq 1$, representing measurement results, such that $a_{1}+\cdots+a_{n}=u$, where $u$ is the unit effect $(u(\alpha)=1$ for all $\alpha \in \Omega)$ and $a_{i}\left(\alpha_{j}\right)=\delta_{i, j}$ for all $i, j=1, \ldots, n$. The information capacity of the system is the maximum size of such a perfectly distinguishable set of states. A complete measurement is a measurement that perfectly distinguishes a maximum

\footnotetext{
${ }^{15}$ We can, of course, regard this as a degenerate category in which there are no morphisms between different objects. My intention, however, is simply to leave it open which mappings between different systems count as processes, as nothing will depend on this. This is another respect in which the approach taken here is "singlesystem".
} 
number of states. In the actual development of their results, they effectively take $\mathcal{M}(A)$ to be the set of complete measurements in this sense.

Masanes and Mueller's Subspace Axiom Here is how Masanes and Mueller explain their version of the subspace axiom in [26]:

Postulate 2 (Equivalence of subspaces). Let $S_{N}$ and $S_{N-1}$ be systems with capacities $N$ and $N-1$, respectively. If $E_{1}, \ldots, E_{N}$ is a complete measurement on $S_{N}$, then the set of states $\omega \in S_{N}$ with $E_{N}(\omega)=0$ is equivalent to $S_{N-1}$.

The notion of equivalence needs some discussion. Postulate 2 states the equivalence of $S_{N-1}$ and

$$
S_{N-1}^{\prime}:=\left\{\omega \in S_{N} \mid E_{N}(\omega)=0\right\} .
$$

Denote the real linear space which contains $S_{N}$ by $V_{N}$; define $V_{N-1}$ analogously, and set $V_{N-1}^{\prime}:=\operatorname{Span}\left(S_{N-1}^{\prime}\right)$. Equivalence means first of all that there is an invertible linear map $L: V_{N-1} \rightarrow V_{N-1}^{\prime}$ such that $L\left(S_{N-1}\right)=S_{N-1}^{\prime}$. But it also means that transformations $\ldots$ on one of them can be implemented on the other... To be more specific, define $\bar{G}_{N-1}^{\prime}$ as the set of transformations in $S_{N}$ that preserve $S_{N-1}^{\prime} \cdots$

$$
\bar{G}_{N-1}^{\prime}:=\left\{T \in G_{N} \mid T S_{N-1}^{\prime}=S_{N-1}^{\prime}\right\} .
$$

The set of reversible transformations $G_{N-1}^{\prime}$ is defined as the restriction of all these transformations to $S_{N-1}^{\prime} \cdots$

A Reformulation Returning to our own formalism, suppose that $x \in X(A)$, and let

$$
F_{x}:=\{\alpha \in \Omega(A) \mid \alpha(x)=0\} .
$$

Note that this is a face of $\Omega(A)$, corresponding exactly to the restricted state space contemplated in Masanes and Mueller's subspace axiom. If we wish to treat this as the state space of a model in our sense, we must associate a test space with it. The simplest option is to define, for $x \in X$,

$$
\mathcal{M}(A)_{x}=\{E \backslash\{x\} \mid E \in \mathcal{M}(A) \text { with } x \in E\} .
$$

Notice that the outcome-space of $\mathcal{M}(A)_{x}$ is the set of outcomes in $X(A)$ that are distinguishable from $x$; that is, the union of the tests in $\mathcal{M}(A)_{x}$ is the set $\{y \in X(A) \mid y \perp x\}$. In the special case of a quantum model, say $A=A(\mathcal{H})$, this is the right definition, since if $\mathcal{K}=x^{\perp}$ is the subspace of $\mathcal{H}$ orthogonal to a unit vector $x$, then any frame $F \in \mathcal{M}(\mathcal{K})$ extends to a frame $E=F \cup\{x\}$ of $\mathcal{H}$, so that $\mathcal{M}(\mathcal{K})=\mathcal{M}(\mathcal{H})_{x}$.

Any state $\alpha \in F_{x}$ defines a probability weight $\alpha_{x}$ on $\mathcal{M}(A)_{x}$, simply by restricting $\alpha$ to outcomes in $\bigcup \mathcal{M}(A)_{x}$, i.e., to outcomes $y \in X(A)$ with $y \perp x$. The mapping $\alpha \mapsto \alpha_{x}$ is obviously affine, but in general is not injective. For a simple example, consider the "square bit": $\mathcal{M}(A)=\left\{\left\{x, x^{\prime}\right\},\left\{y, y^{\prime}\right\}\right\}$, with $\Omega(A)$ the set of all probability weights thereon. Then $\Omega(A)$ is isomorphic to the unit square in $\mathbb{R}^{2}$ under the mapping $\alpha \mapsto(\alpha(x), \alpha(y))$. The face $F_{x}$ can be identified with the right-hand face of the square, i.e., $F_{x}=\{(0, t) \mid 0 \leq t \leq 1\}$. On the other hand, $\mathcal{M}(A)_{x}$ is the trivial test space $\left\{\left\{x^{\prime}\right\}\right\}$, which has only a single probabilty weight. We will therefore need to assume that $\mathcal{M}(A)_{x}$ is large enough to separate points of $F_{x}$, i.e., that if $\alpha, \beta \in F_{x}$ are distinct, then there exists some $y \perp x$ with $\alpha(y) \neq \beta(y)$. This makes $\alpha \mapsto \alpha_{x}$ an injection, so that we can identify $F_{x}$ with a set of probability weights on $\mathcal{M}(A)_{x}$. 
With this in mind, the following now seems to capture the spirit of Masanes and Mueller's axiom:

Definition B.1 Say that a probabilistic theory $\mathcal{C}$ has the subspace property iff, for every $A \in \mathcal{C}$ and every $x \in X(A)$,

(i) $\mathcal{M}(A)_{x}$ separates points of $F_{x}$,

(ii) The model $A_{x}:=\left(\mathcal{M}(A)_{x}, F_{x}\right)$ belongs to $\mathcal{C}$, and

(iii) every symmetry in $G\left(A_{x}\right)$ extends to a symmetry $g \in G(A)$ with $g x=x$.

As an example, let $A=A(\mathcal{H})$ be the quantum model associated with a Hilbert space $\mathcal{H}$. If $x \in X(\mathcal{H})$ is a unit vector, then $F_{x}$ is the set of density operators $W$ such that $\operatorname{Tr}(W x)=0$, or, equivalently, such that $P W P=W$ where $P$ is the orthogonal projection onto the subspace $\mathcal{K}_{x}=x^{\perp}$ orthogonal to $x$. In this case $\mathcal{M}(A)_{x}$ is the set of orthonormal bases of $\mathcal{K}$, so $\mathcal{M}(A)_{x}=\mathcal{M}(\mathcal{K})$, and $A(\mathcal{H})_{x}=A\left(\mathcal{K}_{x}\right)$.

Lemma B.2 Let $\mathcal{C}$ be a probabilistic theory with the subspace property, in which every model is uniform. Then every model in $\mathcal{C}$ is sharp.

Proof: By induction on rank. Clearly, all models of rank 1 are sharp. Assume the result holds for all models of rank $n$ or lower. Suppose $A$ has rank $n+1$ (with $n>0$ ), and let $x \in X(A)$. Since $n+1>1$, we can find some $y \perp x$. Since $A_{y} \in \mathcal{C}$ has rank $n, A_{y}$ is sharp. Therefore, there exists a unique $\delta_{x} \in F_{y}$ - and hence, a unique $\delta_{x} \in \Omega(A)$ - with $\delta_{x}(x)=1$.

Remark: This is the only use we make of Condition (i) in Definition B.1. If one is content to assume that every model in $\mathcal{C}$ is sharp, this condition can be dispensed with.

When dealing with finite-dimensional probabilistic models, one usually assumes that the statespace $\Omega(A)$ is compact. It is equally natural to suppose that $X(A)$ is compact in the topology inherited from $\mathbf{E}(A)$ - equivalently, in, the coarsest topology making every state $\alpha: X(A) \rightarrow[0,1]$ continuous. This is certainly the case for quantum models, where $X(A)$ is the unit sphere in a finite-dimensional Hilbert space, and can be shown to hold for the test space of "complete measurements" considered (implicitly) in [25].

Let us say that a probabilistic model $A$ is spectral if it is sharp, and every states $\alpha \in \Omega(A)$ has a spectral decomposition $\alpha=\sum_{x \in E} \alpha(x) \delta_{x}$ for some $E \in \mathcal{M}(A)$.

Proposition B.3 Let $\mathcal{C}$ be a probabilistic theory with the subspace property, in which all models are uniform and have compact outcome-spaces. Then every model in $A$ is spectral.

Proof: By induction on the rank of $A \in \mathcal{C}$. Spectrality is trivial for models of rank 1 (which have only a single state). Assume the result holds for all $A \in \mathcal{C}$ having $\operatorname{rank}<n=\operatorname{rank}(A)$. Let $\alpha \in \Omega(A)$. Since $X(A)$ is compact and $\alpha$ is continuous on $X(A), \alpha$ takes its minimum value, $m$, $0 \leq m<1$, at some point $x_{o} \in X(A)$. Thus, $\beta:=\alpha-m \delta_{x_{o}}$ is non-negative on $X(A)$, hence, belongs to $\mathbf{V}(A)_{+}$. Now, $u_{A}(\beta)=1-m$, so $\beta_{1}:=(1-m)^{-1} \beta \in \Omega(A)$, and $\alpha=(1-m) \beta_{1}+m \delta_{x_{o}}$. Since $\beta_{1}\left(x_{o}\right)=0, \beta_{1}$ belongs to the face $F_{x_{o}}:=\left\{\alpha \in \Omega(A) \mid \alpha\left(x_{o}\right)=0\right\}$. Because $\mathcal{C}$ has the subspace property, $A_{x_{o}}$ belongs to $\mathcal{C}$. Since $A_{x_{o}}$ has rank $n-1$, our inductive hypothesis implies that $\beta_{1}=\sum_{y \in F} \beta_{1}(y) \delta_{y}$ for some $F \in \mathcal{M}\left(A_{x}\right)=\mathcal{M}(A)_{x}$, i.e., for some $F=E \backslash\left\{x_{o}\right\}, x_{o} \in E \in \mathcal{M}(A)$. 
But now

$$
\alpha=(1-m) \beta_{1}+m \delta_{x_{o}}=\sum_{y \in F}(1-m) \beta(y) \delta_{y}+m \delta_{x_{o}}
$$

which gives a spectral decomposition for $\alpha$.

Remarks: Subspace axioms are usually paired with a conceptually distinct requirement that all systems of a given information capacity are isomorphic. This immediately rules out any nonclassical theory involving superselection rules, or in which there are more than one kind of "bit". As we do not impose such an isomorphism requirement, we avoid this restriction.

\section{B.2 Subspaces plus symmetry}

In addition to one or another version of subspace postulate, [20, 14, 25, 11] also assume that every model $A$ to carries a preferred group $G(A)$ of symmetries, understood as acting on the state space, which is consistent with our choice of $G(A)$ in the previous section. The first three of the cited papers assume a symmetry postulate requiring that $G(A)$ act transitively on pure states, i.e., on the extreme points of $\Omega(A)$. This is also a consequence of the "purification postulate" used in [11].

Definition B.4 A probabilistic model $A$ is symmetric iff $G(A)$ acts transitively on $X(A)$. If $A$ is sharp and all pure states are of the form $\left\{\delta_{x} \mid x \in X(A)\right\}$, this implies $G(A)$ also acts transitively on pure states.

As an example, let $A=A(\mathcal{H})$ be the quantum model associated with a Hilbert space $\mathcal{H}$. As discussed earlier, this means that $\mathcal{M}(A)$ is the set of orthonormal bases of $\mathcal{H}, X(A)$ is (therefore) the unit sphere of $\mathcal{H}$, and $\Omega(A)$ is the set of density operators on $\mathcal{H}$. If we take $G(A)$ to be the group of unitary operators on $\mathcal{H}$, then $G(A)$ certainly acts transitively on $X(A)$.

Notice that, in this example, $G(A)$ acts transitively also on $\mathcal{M}(A)$. In [30] and elsewhere, a uniform test space with a preferred group of symmetries $G(A)$ is said to be fully symmetric if, for every pair of tests $E, F \in \mathcal{M}(A)$ and every bijection $f: E \rightarrow F$, there exists an element $g \in G(A)$ such that $g x=f(x)$ for every $x \in E$. If $A$ has rank $n$, we can define an ordered test to be an $n$-tuple $\left(x_{1}, \ldots, x_{n}\right) \in X^{n}$ where $\left\{x_{1}, \ldots, x_{n}\right\} \in \mathcal{M}(A)$. Then full symmetry is the condition that $G(A)$ act transitively on ordered tests, where $g\left(x_{1}, \ldots, x_{n}\right)=\left(g x_{1}, \ldots, g x_{n}\right)$.

Lemma B.5 Suppose that $\mathcal{C}$ has the subspace property. If every $A \in \mathcal{C}$ is uniform and symmetric, then every $A \in \mathcal{C}$ is fully symmetric.

Proof: Every symmetric model of rank 1 is trivially fully symmetric. Suppose every model in $\mathcal{C}$ having rank $\leq n$ is fully symmetric, and let $A \in \mathcal{C}$ be a model of rank $n+1$. Let $\left(x_{o}, \ldots, x_{n}\right)$ and $\left(y_{o}, \ldots y_{n}\right)$ be any ordered tests of $A$. By symmetry, we can find some $g_{o} \in G(A)$ such that $g_{o} x_{o}=y_{o}$. Now $\left(y_{1}, \ldots, y_{n}\right)$ and $\left(g_{o} x_{1}, \ldots, g_{o} x_{n}\right)$ are both ordered tests of $A_{y_{o}}$. By our induction hypothesis, $A_{y_{o}}$ is fully symmetric, so there exists some $g_{1} \in G\left(A_{y_{o}}\right)$ with $y_{i}=g_{1} g_{o} x_{i}$ for every $i=1, \ldots, n$. By the subspace property, $g_{1}$ extends to a symmetry $g \in G(A)$ with $g y_{o}=y_{o}$ and $g z=g_{1} z$ for every $z \perp y_{o}-$ in particular, $g g_{o} x_{i}=g_{1} g_{o} x_{i}=y_{i}$ for $i=1, \ldots ., n$. Hence, $g g_{o}$ takes $\left(x_{o}, \ldots, x_{n}\right)$ to $\left(y_{o}, \ldots, y_{n}\right)$. 


\section{B.3 A Strengthened Subspace Postulate}

Since we are considering physical processes $\phi \in \operatorname{Proc}(A)$ that need not be normalization-preserving (in particular, need not be associated with symmetries in $G(A)$ ), the following stronger version of the subspace property seems quite as well-motivated a the weaker version discussed above.

Definition B.6 Let $\mathcal{C}$ be a probabilistic theory in which all systems are sharp. Say that $\mathcal{C}$ has the strong subspace property (SSP) iff, for every system $A \in \mathcal{C}$ and every test $E \in \mathcal{M}(A)$, if $x \in E$, then (i) $A_{x} \in \mathcal{C}$ and (ii) any (reversible) process $\phi \in \operatorname{Proc}\left(A_{x}\right)$ lifts to a (reversible) process in $\operatorname{Proc}(A)$ fixing $\delta_{x}$.

Note that the significant difference here from the subspace property of Definition B.1 is the requirement that all processes on $A_{x}$, and not only symmetries, lift to processes on $A$, which can be taken to be reversible if the original processes are.

Lemma B.7 If $\mathcal{C}$ satisfies the SSP, every model in $\mathcal{C}$ has arbitrary reversible filters.

Proof: A system of rank 1 automatically has arbitrary filters: the only test has a single outcome, $x$, and there is just one state, $\delta_{x}$. The mapping $\delta_{x} \mapsto t_{x} \delta_{x}$ defines a p-reversible positive mapping on $\mathbf{V}(A) \simeq \mathbb{R}$.

Now suppose, for purposes of induction, that every system of rank $<n$ has arbitrary reversible filters. Let $E$ be a test, and let $\phi_{E}: \mathbf{V}(E \backslash\{x\}) \rightarrow \mathbf{V}(E \backslash\{x\})$ be a filter with $\phi\left(\delta_{y}\right)=t_{y} \delta_{y}$ for all $y \in E \backslash\{x\}$. Extend this to $\phi: \mathbf{V}(A) \rightarrow \mathbf{V}(A)$ fixing $\delta_{x}$. Now let $z \in E \backslash\{x\}$. Repeating the argument, find $\psi$ a filter on $A$ with $\psi\left(\delta_{y}\right)=\delta_{y}$ for all $y \in E \backslash\{x\}$ and $\psi\left(\delta_{x}\right)=t_{x} \delta_{x}$ with $t_{x}<1$. (In other words, for $z \in E \backslash\{y\}$, we have $t_{z}=1$ if $z \neq x$ and $t_{x}$ the given value.) Composing, we have a positive mapping with

$$
(\psi \circ \phi)\left(\delta_{z}\right)=\psi\left(t_{z} \delta_{z}\right)=t_{z} \psi\left(\delta_{z}\right)=t_{z} \delta_{z}
$$

for $z \neq x$, and

$$
(\psi \circ \phi)\left(\delta_{x}\right)=\psi\left(\delta_{x}\right)=t_{x} \delta_{x}
$$

Thus, we have arbitrary p-reversible filters.

In [30], it is shown that the existence of arbitrary reversible filters plus full symmetry implies the homogeneity of the state space. So we have

Proposition B.8 Let $\mathcal{C}$ satisfy the SSP. If every $A \in \mathcal{C}$ is symmetric, then for every $A \in \mathcal{C}$, $\mathbf{V}(A)$ is homogeneous.

Proof: By Lemma B.7 above, we have arbitrary reversible filters; by Lemma B.5 in previous subsection, every $A \in \mathcal{C}$ is fully symmetric.

The proofs of Theorem 1 and Corollary 1 imply that, in the presence of a spectral decompostion for states, sharpness, the existence of a weak conjugate, and the existence of arbitrary reversible filters are enough to secure the self-duality of $\mathbf{E}(A)$. Thus, Propositions B.3 and B.8 gives us a third route to Jordan models, to put alongside Corollaries 1 and 2 in the main body of this paper: 
Corollary B.9 Let $\mathcal{C}$ satisfy the SSP. If every model $A$ in $\mathcal{C}$ has a compact outcome-space $X(A)$, is symmetric and has a weak conjugate, then $A$ is homogeneous and self-dual, and hence, a Jordan model.

\section{B.4 Jordan models and the SSP}

The preceding results provide an alternative route from four operationally meaningful axioms - sharpness, symmetry, the SSP and the existence of weak conjugates - to euclidean Jordan algebras. The question remains whether all EJAs arise in this way. In fact, the full symmetry enforced by Lemma B.5 significantly constrains the possibilities.

B.10 Lemma: Let $A=A(\boldsymbol{J})$ be the Jordan model associated with a Jordan algebra $\boldsymbol{J}$. If $A$ is fully symmetric, then $\boldsymbol{J}$ is either simple, or a direct sum of one-dimensional Jordan algebras.

Proof: Suppose $\boldsymbol{J}=\bigoplus_{i=1}^{n} \boldsymbol{J}_{i}$ where $\boldsymbol{J}_{1}, \ldots, \boldsymbol{J}_{n}$ are simple euclidean Jordan algebras, with $n \geq 2$. Let $E_{i}$ be a Jordan frame for $\boldsymbol{J}_{i}$; then $E=\bigcup_{i=1}^{n} E_{i}$ is a Jordan frame for $\boldsymbol{J}$. Let $x_{1} \in E_{1}$ and $x_{2} \in E_{2}$. By full symmetry, there exists a symmetry $g \in G(A)$ such that $g x_{1}=x_{2}, g x_{2}=x_{1}$, and $g y=y$ for all $y \in E \backslash\left\{x_{1}, x_{2}\right\}$. Now let $p_{i}$ be the central projection associated with the $i$-th summand, so that $p_{1}$ and $p_{2}$ are the central covers of (the smallest central projections above) $x_{1}$ and $x_{2}$, respectively. Hence, $g\left(p_{1}\right)$ is the central cover of $g x_{1}=x_{2}$, i.e, $g\left(p_{1}\right)=p_{2}$, and similarly $g p_{2}=p_{1}$. But now if $y \in E_{1}$ is any point other than $x_{1}$, we have $y=g y \leq g p_{1}=p_{2}$, which are impossible, as $y$ is orthogonal to $p_{2}$. So $E_{1}=\left\{x_{1}\right\}$, and $\boldsymbol{J}_{1}$ is one-dimensional. Since $\boldsymbol{J}_{1}$ is arbitrary, all summands are one-dimensional.

Thus, a probabilistic theory satisfying the hypotheses of Corollary B.9 will comprise only simple Jordan models and classical systems. In particular, such a theory cannot accommodate superselection rules. This is also true, however, of earlier reconstructions based on versions of the subspace axiom that assume all systems of the same information capacity to be isomorphic. (If the theory is monoidal, roughly meaning that it allows for the formation of composite systems and meaning more precisely that the category in question has a symmetric monoidal structure, satisfying some reasonable constraints on how this interacts with the theory's probabilisic apparatus; see [9] for details - then there are further constraints. These matters are discussed in [5]. For a discussion of how monoidal "process theories" in the sense of $[1,13]$ can be represented as probabilistic theories in the sense of this paper, see [32].)

We now show that the assumptions discussed in this Appendix imply no further constraints on Jordan models. To this end, it will be enough to exhibit a probabilistic theory $\mathcal{C}$ containing all simple Jordan models, and assigning to each such model $A$ a monoid $\operatorname{Proc}(A)$ of allowed processes in such a way that $A$ is symmetric and $\mathcal{C}$ enjoys the SSP.

Proposition B.11: Let $\mathcal{C}$ be the probabilistic theory consisting of all full, simple euclidean Jordan models, with processes on a given model $A(\boldsymbol{J})$ consisting of composites of maps of the form $U_{a}$, $a \in J^{16}$. Then every model in $\mathcal{C}$ is symmetric, and satisfies the SSP.

\footnotetext{
${ }^{16}$ The structure group, $\Gamma(\boldsymbol{J})$, of a euclidean Jordan algebra $\boldsymbol{J}$ consists of all non-singular positive mappings $\phi: \boldsymbol{J} \rightarrow \boldsymbol{J}$ such that $U_{\phi}(a)=\phi U_{a} \phi^{*}$ for every $a \in \boldsymbol{J}$. A theorem of Koecher [23] shows that the connected component of the identity in $\Gamma(\boldsymbol{J})$ consists exactly of composites of mappings of the form $U_{a}$. In other words, in this theory, $G(A)$ is precisely the connected identity component of $\Gamma(\boldsymbol{J})$.
} 
Proof: Let $A=A(\boldsymbol{J})=(\mathcal{M}(\boldsymbol{J}), \Omega(\boldsymbol{J}))$ be the Jordan model associated with an EJA $\boldsymbol{J}$. Thus, $\mathcal{M}(\boldsymbol{J})$ is the set of Jordan frames, and $X(\boldsymbol{J})$ the set of primitive idempotents, of $\boldsymbol{J}$. If $\boldsymbol{J}$ is simple, symmetry (indeed, full symmetry) of $A$ under $G(A)$ follows from ([18], IV.2.5) ${ }^{17}$ It follows easily that $G(A)$ acts transitively on Jordan frames even if $\boldsymbol{J}$ is not simple (since each such frame is the disjoint union of frames chosen from each simple summand). Let $p \in P(\boldsymbol{J})$. Then ([2], Prop. 1.38, Lemma 1.39, Prop. 1.43, Prop. 2.32 and remarks preceding latter), $\boldsymbol{J}_{p}:=U_{p}(\boldsymbol{J})$ is a hereditary Jordan subalgebra, meaning that if $0 \leq a \leq b \in \boldsymbol{J}_{p}$, then $a \in \boldsymbol{J}_{p}$ as well. The unit of $\boldsymbol{J}_{p}$ is $p$. It is important to note here that $\left(\boldsymbol{J}_{p}\right)_{+}=\boldsymbol{J}_{+} \cap \boldsymbol{J}_{p}$ (that is, an element of $\boldsymbol{J}_{p}$ is positive in $\boldsymbol{J}_{p}$ iff it is positive in $\boldsymbol{J}$ ). This follows from spectral theory; see [2] Prop. 1.22 and subsequent discussion. It follows that if $a, b \in \boldsymbol{J}_{p}, a \leq b$ in $\boldsymbol{J}_{p}$ iff $a \leq b$ as elements of $\boldsymbol{J}$. One can also show ([2], Lemma 1.45) that if $a \in \boldsymbol{J}_{p}$ and $b \in \boldsymbol{J}_{p^{\prime}}$, then $a \bullet b=0$.

Now let $e$ be a primitive idempotent in $\boldsymbol{J}$ that happens to lie in $\boldsymbol{J}_{p}$ : then $e$ is an idempotent in $\boldsymbol{J}_{p}$ as well. If $f$ is another nonzero idempotent in $\boldsymbol{J}_{p}$ with $f \leq e$, then $f$ is still idempotent in $\boldsymbol{J}$ and, by remarks above, $f \leq e$ in $\boldsymbol{J}$, whence, as $e$ is minimal, $e=f$. Thus, $e$ is primitive in $\boldsymbol{J}_{p}$. Conversely, let $e$ be primitive idempotent in $\boldsymbol{J}_{p}$. Then $e$ is still idempotent in $\boldsymbol{J}$. If $f$ is an idempotent of $\boldsymbol{J}$ with $0<f \leq e$ in $\boldsymbol{J}$, then $f \in \boldsymbol{J}_{p}$, since the latter is hereditary. As $e$ is primitive in $\boldsymbol{J}_{p}, f=e$. Thus, $e$ is primitive in $\boldsymbol{J}$.

This shows that $X\left(\boldsymbol{J}_{p}\right)=\boldsymbol{J}_{p} \cap X(\boldsymbol{J})$. It follows that $\mathcal{M}\left(\boldsymbol{J}_{p}\right)$ is the set of all sets of primitive idempotents of $\boldsymbol{J}$ summing to $p$. Now let $p=u-x$ where $x$ is a primitive idempotent of $\boldsymbol{J}$. Then $F \in \mathcal{M}\left(\boldsymbol{J}_{p}\right)$ iff $F \cup\{x\} \in \mathcal{M}(\boldsymbol{J})$. In other words, $\mathcal{M}\left(\boldsymbol{J}_{p}\right)=\mathcal{M}(A)_{x}$ in the notation of Definition B.1.

It remains to show that any (p-reversible) process in $\operatorname{Proc}\left(A_{x}\right)$ extends to a (reversible) process in $\operatorname{Proc}(A)$ leaving $x$ fixed. It suffices to show this holds for processes of the form $U_{a}$, with $a \in \boldsymbol{J}_{p}$. Since $x \bullet b=b \bullet x=0$ for all $b \in \boldsymbol{J}_{p}$, a straightforward calculation then shows that $U_{a+x} b=U_{a} b$ for all $b \in \boldsymbol{J}_{p}$, and that $U_{a+x} x=x$. Hence, $U_{a+x}$ is the desired extension of $U_{a}$. Note that if $U_{a}$ is invertible, then $a$ is invertible in $J_{p}$. By Lemma A.4 (b), there exists some $b \in \boldsymbol{J}_{p}$ with $a \bullet b=p$ and $a^{2} \bullet b=a$. Since $x \bullet b=0$ for all $b \in \boldsymbol{J}_{p}$, we have

$$
(a+x) \cdot(b+x)=a \cdot b+x^{2}=a \cdot b+x=p+x=u,
$$

and

$$
(a+x)^{2} \cdot(b+x)=\left(a^{2}+x\right) \cdot(b+x)=a^{2} \cdot b+x^{2}=a+x .
$$

Invoking Lemma A.4 (b) again, this implies that $b+x$ is the inverse of $a+x$ in $\boldsymbol{J}$, whence, by Lemma A.4 (c), $U_{a+x}$ is invertible.

\footnotetext{
${ }^{17}$ Or, more exactly, from the proof thereof. For any two Jordan-orthogonal primitive idempotents $x, y$ there exists a symmetry - an element $s \in A$ with $s^{2}=u$ - such that $U_{s}(x)=y$ ([18], IV.2.4). This, plus an induction, allows one to construct an automorphism of the form $U_{a}$ taking one Jordan frame to another.
} 